\title{
Fibroblast Activation Protein Regulates Lesion Burden and the Fibroinflammatory Response in Apoe-Deficient Mice in a Sexually Dimorphic Manner

James Monslow, ${ }^{*}$ Leslie Todd, ${ }^{*}$ John E. Chojnowski, ${ }^{*}$ Priya K. Govindaraju, ${ }^{*}$ Richard K. Assoian, ${ }^{\dagger}$ and Ellen Puré

From the Departments of Biomedical Sciences* and Systems Pharmacology and Translational Therapeutics, ${ }^{\dagger}$ University of Pennsylvania, Philadelphia Pennsylvania

Accepted for publication January 2, 2020.

Address correspondence to James Monslow, Ph.D., Hill Pavilion 430; or Ellen Puré, Ph.D., Hill Pavilion 410E, University of Pennsylvania, $380 \mathrm{~S}$ University Ave., Philadelphia, PA 19104. E-mail: jmonslow@ vet.upenn.edu or epure@upenn. edu.

\begin{abstract}
Fibroblast activation protein (FAP) has been established as an inducible and mesenchymal cell-specific mediator of disease progression in cancer and fibrosis. Atherosclerosis is a fibroinflammatory disease, and FAP was previously reported to be up-regulated in human atherosclerotic plaques compared with normal vessel. We investigated the spatial and temporal distribution of Fap-expressing cells in a murine model of atherosclerosis and used a genetic approach to determine if and how Fap affected disease progression. Fap was found to be expressed predominantly on vascular smooth muscle cells in lesions of athero-prone Apoe $\mathrm{C}^{-I^{-}}$mice. Global deletion of Fap $\left(\mathrm{Fap}^{-{ }^{-}}\right)$in $\mathrm{Apoe}^{-I^{-}}$mice accelerated atherosclerotic disease progression in both males and females, with the effect observed earlier in males. Sexspecific effects on lesion morphology were observed. Relative levels of extracellular matrix, fibrotic, and inflammatory cell content were comparable in lesions in male mice regardless of Fap status. In contrast, lesions in $\mathrm{Fap}^{-/-}$female mice were characterized by a more fibrotic composition due to a reduction in inflammation, specifically a reduction in Mox macrophages. Combined, these data suggest that Fap restrains the progression of atherosclerosis and may contribute to the sexually dimorphic susceptibility to atherosclerosis by regulating the balance between inflammation (an indicator of vulnerability to plaque rupture) and fibrosis (an indicator of plaque stability). (Am J Pathol 2020, 190: 1118-1136; https://doi.org/10.1016/j.ajpath.2020.01.004)
\end{abstract}

Cardiovascular disease (CVD) refers to a multitude of cardiac and vascular complications most often caused by coronary arterial disease, stroke, and peripheral vascular disease. CVD caused by atherosclerosis remains the main cause of death in the United States. ${ }^{1}$ CVD, particularly atherosclerosis, is a disease of aging ${ }^{2,3}$ characterized by the formation of neointima (lesion), a highly integrated process that involves endothelium, vascular smooth muscle cells (VSMCs), inflammatory cell recruitment, and lipid metabolism. ${ }^{4}$ The mechanisms responsible for these processes are multifaceted and often interdependent. Furthermore, the net effect of these mechanisms is directly affected by environmental factors, notably diet and exercise, ${ }^{5}$ as well as aging-related cholesterol and environmental-independent factors, such as arterial stiffening. 6,7

Recent studies have indicated that increased fibrillar collagen and vascular stiffness stimulate endothelial permeability, leukocyte transmigration, and macrophage adhesion to subendothelial extracellular matrix (ECM), ${ }^{8-10}$ thus establishing an important link between the stromal or mesenchymal and early inflammatory components of atherosclerosis. Ongoing crosstalk between cells and ECM in these compartments affects the progressing size, composition, and architecture of lesions and is thus directly implicated in the clinical outcome. A paradigm exists that suggests that the balance between lesional inflammation and fibrosis is a critical determinant of plaque stability and in fact is a better indicator of clinical sequelae than lesion size. ${ }^{11}$ Specifically, the formation of VSMC- or ECM-rich

Supported by NIH grants HL138906 (E.P.) and AG047373 (R.K.A.) and National Science Foundation Science and Technology Center for Engineering Mechanobiology Award CMMI-1548571 (R.K.A.).

Disclosures: None declared. 
lesions, characterized by high collagen content, thick fibrotic caps, and a paucity of inflammation, correlates with plaque stabilization. Conversely, robust lesional inflammation, coupled with low to modest fibrosis, is associated with increased susceptibility to plaque rupture. Targeting pathways central to ECM modification that tip this balance toward the former may therefore lead to the development of new therapeutics to combat the remodeling of atherosclerotic lesions that promotes plaque rupture and the likelihood of myocardial infarction.

ECM remodeling, in particular that of fibrillar collagen, is a hallmark of CVD. The biosynthetic pathways of collagen are well defined, yet the steps involved in its degradation and turnover are less well understood. Atherosclerotic lesions contain elevated levels of collagenases derived from resident VSMCs and recruited inflammatory cells. These collagenases, which are secreted [matrix metallopeptidase (MMP) 1, MMP-8, and MMP-13] or membrane bound (MMP-14), have complex roles in lesion formation and stability because of their overlapping and opposing effects. Collagenase overactivity can lead to severe tissue destruction (as opposed to controlled ECM breakdown), ${ }^{12}$ increase macrophage invasion, ${ }^{13-15}$ and promote angiogenesis, ${ }^{15}$ thus tipping the balance toward inflammation and plaque instability. In contrast, MMP-2, -9 , and -14 (all of which have gelatinase activity) promote VSMC proliferation and migration, which may in turn have the net effect of enhancing fibrotic cap formation and lesion stability. ${ }^{16}$

Fibroblast activation protein (FAP) is a serine protease involved in the ordered proteolytic processing of collagen. A member of the dipeptidyl peptidase family, FAP is expressed at low to undetectable levels under homeostatic conditions ${ }^{17-23}$ but up-regulated on cells involved in ECM remodeling in a myriad of pathophysiologic and pathologic conditions, including wound repair, fibrosis, and inflammation. ${ }^{18,19,24,25}$ It has also been well characterized in multiple tumor types, playing critical roles in tumorigenesis by influencing tumor cell growth, intratumoral desmoplasia, angiogenesis, and immunosuppression. ${ }^{17,26-32}$ Interestingly, two separate studies revealed that FAP was present in human atherosclerotic plaques but not normal nonatherosclerotic vascular tissue. ${ }^{33,34}$ Furthermore, lesional FAP content increased with plaque severity, and FAP expression was suggested to be limited to VSMCs. ${ }^{33}$

VSMCs represent a heterogeneous population in atherosclerosis. Three major populations of VSMCs have been identified based on their spatial and temporal distributions and phenotype. ${ }^{11}$ First, medial differentiated VSMCs in the vessel wall express $\alpha$-smooth muscle actin (Acta2; as well as other differentiation markers, such as smooth muscle myosin heavy chain and transgelin) under homeostatic conditions. During the early stages of atherogenesis at sites of disturbed flow (high vascular curvature and branch points) and endothelial dysfunction, medial differentiated VSMCs transition to a dedifferentiated state. Second, this transition to lesional dedifferentiated VSMCs is characterized by a phenotypic switch characterized by down-regulation of Acta2 expression and often, but not always, a concomitant induction of vascular cell adhesion molecule 1 (Vcam1) expression. Lesional dedifferentiated VSMCs are also more proliferative, produce and remodel neointimal matrix, and secrete inflammatory mediators. Third, fibrotic caps, which are prominent in more stable lesions are enriched in lesional fibrotic VSMCs that express high levels of Acta2 and little to no Vcam1 (similar to media differentiated VSMCs) yet are also proliferative and produce fibrotic matrix components. ${ }^{35-37}$ We hypothesized that FAP may be differentially expressed on these various VSMC subpopulations and may have a potential role in CVD, in particular in regulating the fibroinflammatory response and matrix remodeling characteristics of atherosclerotic lesions.

The aim of this study was to establish the spatial and temporal features of Fap expression in the apolipoprotein E-null $\left(\right.$ Apoe $\left.^{-/-}\right)$mouse model of atherosclerosis to have an in vivo correlate to human CVD in which to study Fap. Interestingly, Fap was identified only on lesional dedifferentiated mesenchymal cells, purportedly VSMCs. Moreover, by genetically deleting Fap in Apoe $e^{-/-}$mice, a role for Fap in atherosclerotic disease progression was discovered, showing that Fap contributes to the balance between inflammation and fibrosis in a sex-dependent manner that has implications for plaque stability.

\section{Materials and Methods}

\section{Animals}

Wild-type C57BL/6 mice for in vitro experiments were purchased from Charles River Laboratories (Wilmington,

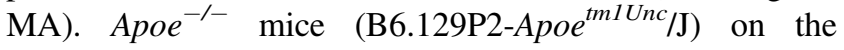
C57BL/6J background were purchased from The Jackson Laboratory (Bar Harbor, ME). Fap ${ }^{-1-}$ mice (Fap lacZ/acZ) were provided by Boehringer Ingelhiem International $\mathrm{GmbH}$ (Ingelheim am Rhein, Germany) ${ }^{38}$ and backcrossed at least 11 generations with $\mathrm{C} 57 \mathrm{BL} / 6 \mathrm{~J}$ mice and then to B6.129P2-Apoe $e^{t m 1 U n c} / \mathrm{J}$ to generate homozygous congenic Apoe $^{-/-}$. Fap ${ }^{-1-}$ mice. All mice were housed in University of Pennsylvania facilities, and all research was overseen by University Laboratory Animal Resources. Experimental protocols were approved by the Institutional Animal Care and Use Committee and in compliance with the NIH's Guide for the Care and Use of Laboratory Animals. ${ }^{39}$

\section{Serum Collection and Metabolite Analysis}

Blood was collected by cardiac perfusion after euthanasia. Blood was transferred directly into BD Microtainer Serum Separator Tubes (BD Biosciences, San Jose, CA) and left to stand for 30 minutes. Tubes were then centrifuged for 5 minutes at $6000 \times g$, serum (upper solution) was collected, and cholesterol and triglyceride analysis was performed by 
Table 1 Antibody Resource List

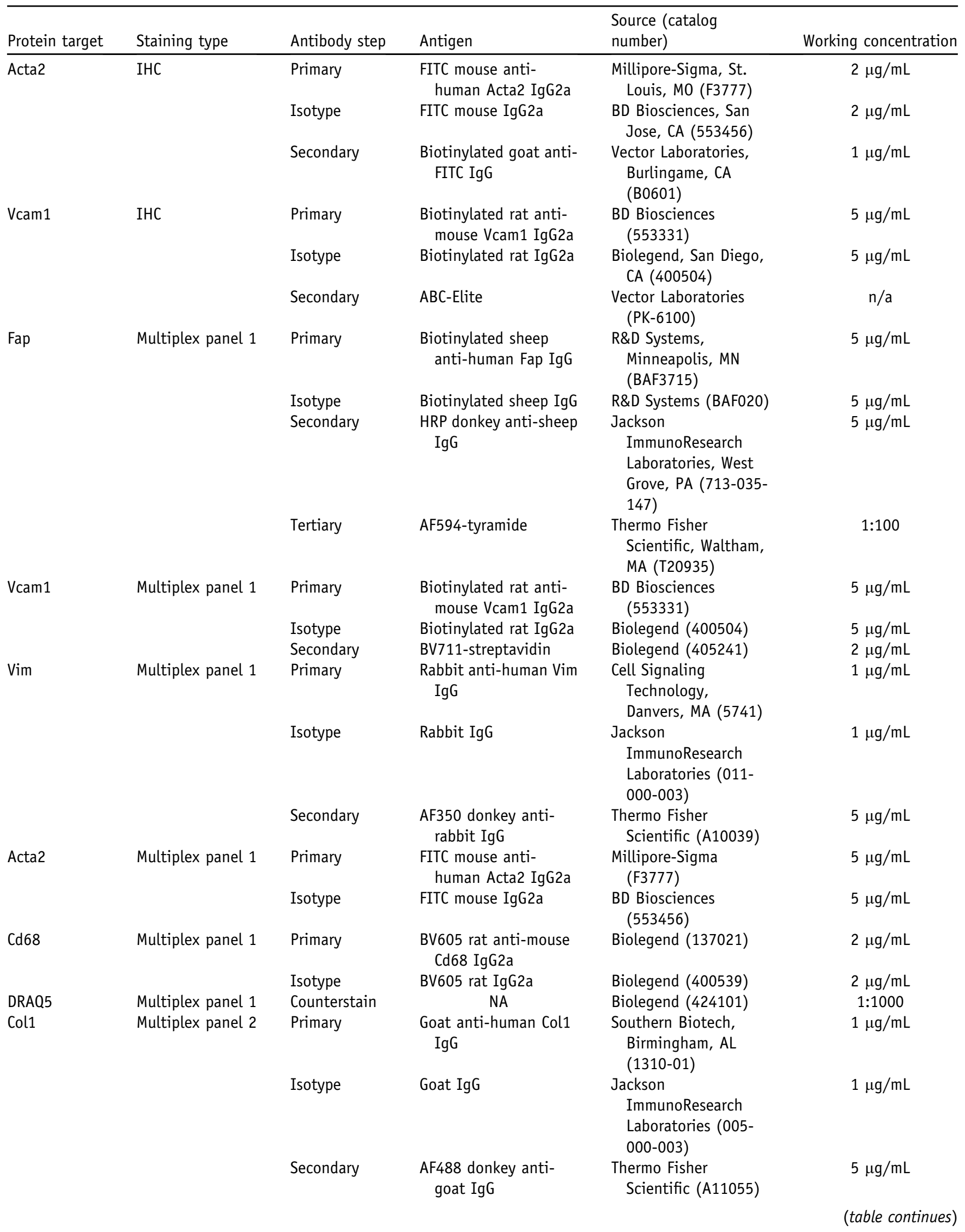


Table 1 (continued)

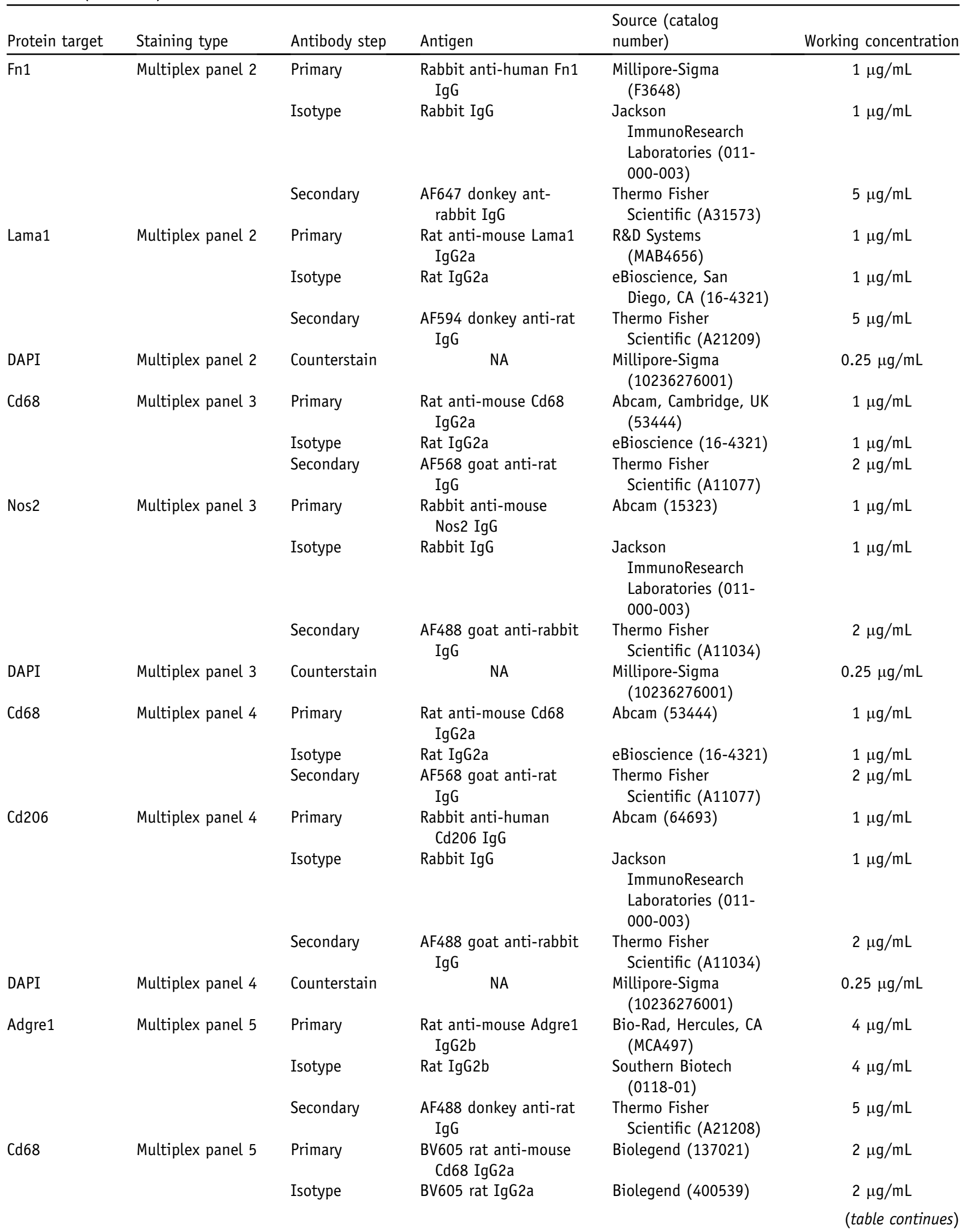


Table 1 (continued)

\begin{tabular}{|c|c|c|c|c|c|}
\hline Protein target & Staining type & Antibody step & Antigen & $\begin{array}{l}\text { Source (catalog } \\
\text { number) }\end{array}$ & Working concentration \\
\hline \multirow[t]{2}{*}{ Hmox1 } & Multiplex panel 5 & Primary & $\begin{array}{l}\text { Rabbit anti-human } \\
\text { Hmox1 IgG }\end{array}$ & Abcam (68477) & $0.5 \mu \mathrm{g} / \mathrm{mL}$ \\
\hline & & Isotype & Rabbit IgG & $\begin{array}{l}\text { Jackson } \\
\text { ImmunoResearch } \\
\text { Laboratories (011- } \\
\text { 000-003) }\end{array}$ & $0.5 \mu \mathrm{g} / \mathrm{mL}$ \\
\hline DAPI & Multiplex panel 5 & Counterstain & NA & $\begin{array}{l}\text { Millipore-Sigma } \\
\quad(10236276001)\end{array}$ & $0.25 \mu \mathrm{g} / \mathrm{mL}$ \\
\hline
\end{tabular}

Acta2, $\alpha$-smooth muscle actin; Adgre1, adhesion G protein-coupled receptor E1; Col1, collagen type I; Fap, fibroblast activation protein; FITC, fluorescein isothiocyanate; Fn1, fibronectin; Hmox1, heme oxygenase; IHC, immunohistochemistry; Lama1, laminin; NA, not applicable; Nos2, nitric oxide synthase 2; Vcam1, vascular cell adhesion molecule 1; Vim, vimentin.

the Mouse Phenotyping, Physiology and Metabolism Core at the UPenn Diabetes Research Center.

\section{Analysis of Aortic Atherosclerotic Lesion Burden}

Atherosclerotic lesion burden of the whole aorta was performed as previously described. ${ }^{35,40}$ Briefly, the vasculature was perfused with $10 \mathrm{~mL}$ of magnesium- and calcium-free phosphate-buffered saline (PBS). Aortas (the entire length from the heart to the branching femoral arteries) were stripped of perivascular adipose tissue and dissected. Tissues were washed briefly in PBS and 60\% isopropanol, stained with Oil Red O solution (60\% solution, MilliporeSigma, St. Louis, MO) for 10 minutes at room temperature, and washed again with $60 \%$ isopropanol followed by distilled water. Any remaining perivascular adipose tissue was then removed under a dissecting microscope before the aortas were filleted to expose the luminal surface. Aortas were placed luminal side up on dental wax sheets and kept hydrated, and atherosclerotic lesions were photographed using a standard digital camera.

\section{Histochemical Staining for Atherosclerotic Lesion Burden and Morphology in the Aortic Root}

Mouse hearts were embedded in OCT medium, and $10-\mu \mathrm{m}$ serial cross-sections of the entire aortic root (approximately $480 \mu \mathrm{m})$ were cut and mounted on positively charged microscope slides. For analysis of atherosclerotic lesion burden, six sections, equally spaced across the entire aortic root, were stained with hematoxylin-2 and eosin-Y (Richard Allen Scientific, Thermo Fisher Scientific, Waltham, MA) following manufacturer's protocols. For histochemical analysis of lesion morphology, three serial cross-sections per root were analyzed; Oil Red $\mathrm{O}$ was used to visualize lipid content, a modified Masson's Trichrome (omitting the nuclear dye, Millipore-Sigma) was used for total collagen, and Picrosirius Red (Millipore-Sigma) was used for fibrillar collagen, as per manufacturers' protocols. Sections stained with hematoxylin and eosin, Masson's Trichrome, and Picrosirius Red were dehydrated and hard-mounted in Cytoseal-60 (Richard Allen Scientific). Oil Red O-stained sections were aqueous-mounted in SlowFade Gold (Thermo Fisher Scientific).

\section{Immunostaining for Atherosclerotic Lesion} Morphology in the Aortic Root

Antibodies used to examine lesion morphology are listed in Table 1. Immunohistochemical staining was used to separately analyze Acta2 and Vcam1 content. Sections were fixed in acetone for 15 minutes at $-20^{\circ} \mathrm{C}$. Sections were consecutively treated to block endogenous peroxidase (3\% hydrogen peroxide for 15 minutes) with $10 \%$ normal serum blocking solution [dependent on host of secondary antibody, in $1 \%$ bovine serum albumin (BSA)/ PBS for 15 minutes] and endogenous biotin (streptavidinbiotin blocking kit, Vector Laboratories, Burlingame, CA). Sections were then incubated with the indicated primary antibody or IgG isotype control in blocking solution overnight at $4^{\circ} \mathrm{C}$ (Table 1). Where required, sections were then incubated with biotinylated-IgG secondary antibody (specific to host of primary antibody) diluted in $1 \%$ BSA/PBS for 1 hour at room temperature. Sections were then incubated with streptavidin-horseradish peroxidase diluted in $1 \% \mathrm{BSA} / \mathrm{PBS}$ for 30 minutes at room temperature, equilibrated in sterile water for $5 \mathrm{mi}$ nutes, and then developed with the diaminobenzidine substrate kit (Dako, Agilent, Santa Clara, CA). Samples were counterstained with hematoxylin, dehydrated, and mounted in Cytoseal-60.

Immunofluorescent multiplex staining was used to analyze all other proteins (Table 1). Multiplex panel 1 was used to determine Fap lesional localization, and sections were costained for Fap, Vcam1, vimentin (Vim), Acta2, and Cd68 and counterstained with DRAQ5 nuclear stain. Multiplex panel 2 was used to co-stain for the ECM proteins collagen type I (Col1), fibronectin (Fn1), and laminin (Lama1). 


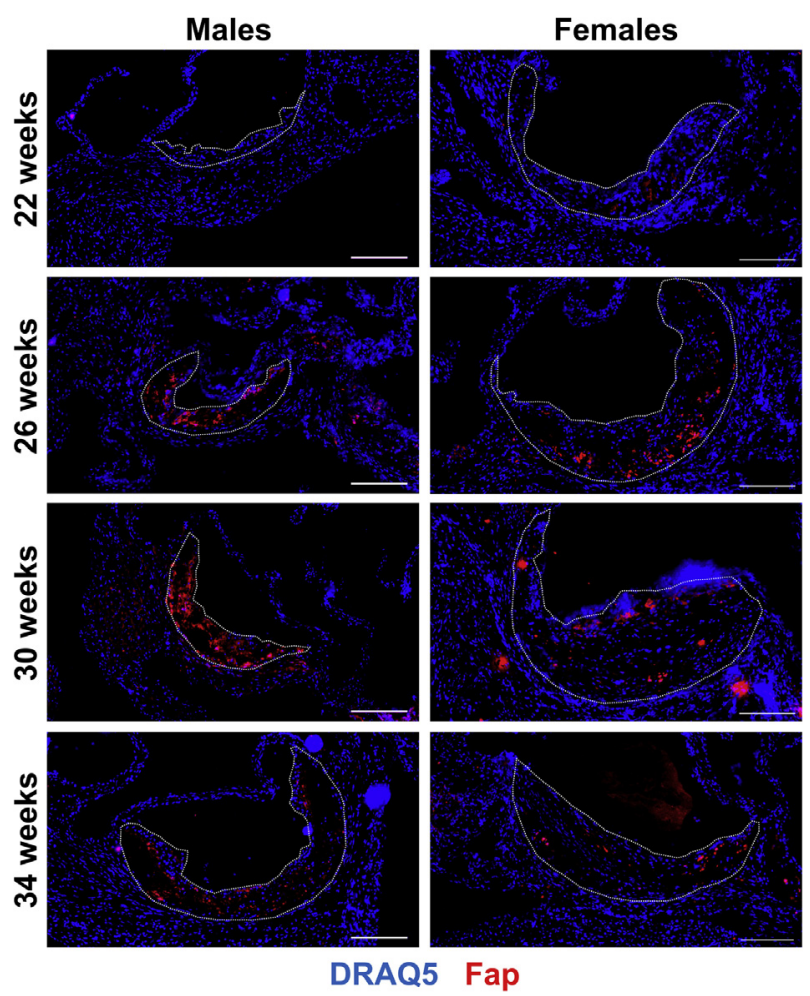

Figure 1 Spatiotemporal expression of fibroblast activation protein (Fap) in atherosclerotic lesions. Male and female $\mathrm{Apoe}^{-/-}$mice were maintained on a chow diet and aged to $22,26,30$, or 34 weeks. Then $10-\mu \mathrm{m}$ frozen sections of aortic roots were co-stained with antibodies targeted to Fap (red) and counterstained with DRAQ5 nuclear stain (blue). Atherosclerotic lesions are highlighted with dotted lines. Scale bars $=200 \mu \mathrm{m}$.

Multiplex panel 3 was used to stain M1 macrophages for Cd68 and nitric oxide synthase 2 (Nos2) (counterstained with DAPI nuclear stain). Multiplex panel 4 was used to stain M1 macrophages for $\mathrm{Cd} 68$ and $\mathrm{Cd} 206$ (counterstained with DAPI). Finally, multiplex panel 5 was used to stain Mox macrophages for $\mathrm{F} 4 / 80$ [adhesion $\mathrm{G}$ protein-coupled receptor E1 (Adgre1)], Cd68, and heme oxygenase (Hmox1). Sections were first fixed in acetone (as described above), then $10 \%$ normal blocking solution (3\% hydrogen peroxide/ endogenous biotin blocking steps were also added for multiplex panel 1 for Fap). Sections were then incubated with the desired primary antibody or IgG isotype control in blocking solution overnight at $4{ }^{\circ} \mathrm{C}$. Where required, sections were then treated with immunofluorescent-labeled secondary antibodies [horseradish peroxidase-secondary antibody, then fluorescent-tyramide (Thermo Fisher Scientific) for Fap], counterstained for nuclei, and then aqueous mounted in SlowFade Gold.

\section{Isolation of Primary Murine VSMCs}

Primary murine VSMCs were harvested from 8- to 12-week-old wild-type C57BL/6 male mice using the partial-digest method. Descending thoracic aortas were isolated and pooled (groups of 5) into $10 \mathrm{~mL}$ of ice-cold Hanks' balanced salt solution (HBSS). Aortas were digested in $20 \mathrm{mg} / \mathrm{mL}$ of type II collagenase (Worthington Biochemical Corp., Lakewood, NJ) in HBSS for 10 minutes at $37^{\circ} \mathrm{C}$, then rinsed in HBSS. The adventitia from each aorta was removed using a dissecting microscope. The remaining vascular tissue was chopped with dissecting scissors then partially digested in $10 \mathrm{~mL}$ of $20 \mathrm{mg} / \mathrm{mL}$ of type II collagenase $/ 10 \mathrm{mg} / \mathrm{mL}$ of elastase (Worthington Biochemical Corp.) in HBSS for an additional 20 minutes at $37^{\circ} \mathrm{C}$. A total of $10 \mathrm{~mL}$ of VSMC medium [Dulbecco's modified Eagle's medium with $4.5 \mathrm{~g} / \mathrm{L}$ of glucose, $584 \mathrm{mg} /$ L of L-glutamine, $110 \mathrm{mg} / \mathrm{L}$ of sodium pyruvate (Corning, Corning, NY), supplemented with $20 \mathrm{IU}$ of penicillin, 20 $\mu \mathrm{g} / \mathrm{mL}$ of streptomycin (Corning), $50 \mu \mathrm{g} / \mathrm{mL}$ of gentamicin (Thermo Fisher Scientific), and 10\% fetal calf serum (Gemini Bio-Products, West Sacramento, CA)] was added, and the tissue fragments were collected by centrifugation at $250 \times g$ for 5 minutes at $4^{\circ} \mathrm{C}$. The supernatant was discarded and the cells and tissue fragments gently resuspended in $2 \mathrm{~mL}$ of VSMC medium and plated into two $35-\mathrm{mm}$ dishes. Cells were maintained in a humidified incubator with $5 \%$ carbon dioxide for 48 hours at $37^{\circ} \mathrm{C}$. An additional $1 \mathrm{~mL}$ of VSMC medium was then added per dish,

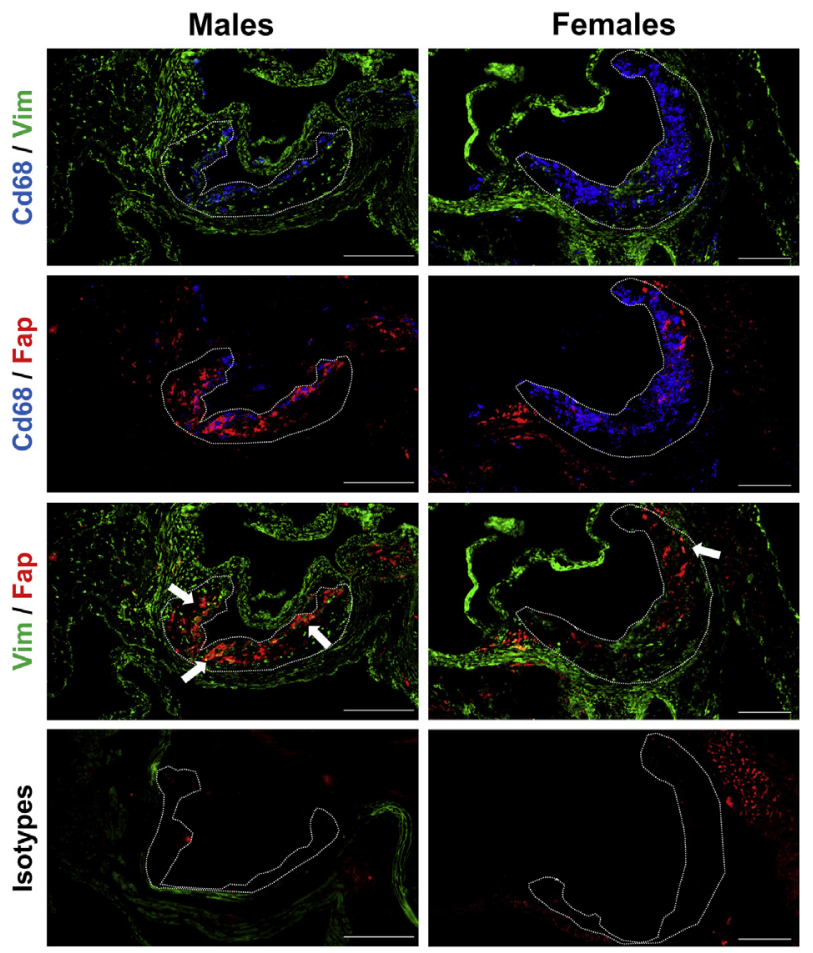

Figure 2 Fibroblast activation protein (Fap) expression is localized to mesenchymal cell-rich but not inflammatory cell-rich regions of atherosclerotic lesions. Male and female $A p o e^{-/-}$mice were maintained on a chow diet and aged to 26 weeks. Then $10-\mu \mathrm{m}$ frozen sections of aortic roots were co-stained with antibodies targeted to inflammatory regions [CD68 (blue)], mesenchymal regions [vimentin (Vim) (green)], and Fap (red) or isotype IgG controls. Atherosclerotic lesions are highlighted with dotted lines. Arrows indicate Fap/Vim overlapping regions (yellow). Scale bars $=200 \mu \mathrm{m}$. 
and the cells were incubated for an additional 5 to 7 days before passaging. For cells at passage 0 to $1,250 \mu \mathrm{g} / \mathrm{mL}$ of amphotericin B (Thermo Fisher Scientific) was supplemented to the growth medium. Cells were typically passaged at $80 \%$ to $90 \%$ confluence at a ratio of $1: 2$. Cells were used for experiments at passage 2 to 3 .

\section{Analysis of VSMC Phenotype in Vitro by Immunostaining and Quantitative PCR}

To examine VSMC phenotype by immunostaining, $2 \times 10^{5}$ of VSMCs were plated onto type I collagen-coated coverslips $(50 \mu \mathrm{g} / \mathrm{mL})$ in $35-\mathrm{mm}$ dishes in $2 \mathrm{~mL}$ of VSMC medium for 48 hours. VSMC medium was then replenished $\pm 2 \mathrm{ng} / \mathrm{mL}$ recombinant human transforming growth factor$\beta 1$ (rhTGF- $\beta 1$ ) for an additional 48 hours. Phase contrast images were captured, and samples were fixed in $4 \%$ paraformaldehyde/PBS for 30 minutes at room temperature. Cells were permeabilized with $0.1 \%$ Triton X-100/1\% BSA/ PBS for 10 minutes at room temperature, then stained with antibodies for Acta2 and Vcam1 as described above. After counterstaining with DAPI, the coverslips were aqueous mounted in SlowFade Gold onto coverslip glass-bottomed 60-mm dishes for imaging.

To assess gene expression by quantitative PCR, $2 \times 10^{5}$ VSMCs were plated into $35-\mathrm{mm}$ dishes in normal VSMC medium for 48 hours. VSMC medium was then replenished $\pm 2 \mathrm{ng} / \mathrm{mL}$ rhTGF- $\beta 1$ for an additional 48 hours. The supernatant was removed, and the cells washed once in PBS and then harvested in $1 \mathrm{~mL}$ of TRIzol (Thermo Fisher Scientific). Total RNA was purified following the manufacturers' protocol. A total $1 \mu \mathrm{g}$ RNA was reverse transcribed using the TaqMan Reverse Transcription kit (Applied Biosystems, Thermo Fisher Scientific). Quantitative PCR was run using SYBR green reagent (Thermo Fisher Scientific) and the relative standard curve method. Fourfold serial dilutions ranging from 64 to $0.25 \mathrm{ng}$ of cDNA per well were run in $12.5-\mu \mathrm{L}$ reactions in triplicate per gene target (with the exception of Fap, 256 to $1 \mathrm{ng}$ ). The following primer pairs were used for each gene target: Acta2 (forward: 5'-CCAGAGCAAGAGAGGGATCCT-3' and reverse: $5^{\prime}$-TGTCGTCCCAGTTGGTGATG-3'), Fap (forward: 5'-CACCTGATCGGCAATTTGTG-3' and reverse: 5'-CCCATTCTGAAGGTCGTAGATGT-3'), Gapdh (forward: $5^{\prime}$-GACGGCCGCATCTTCTTGT-3' and reverse: 5'-CACACCGACCTTCACATTTT-3'), Myocd (forward: 5'-CAAACTGGTGTTTCTTCTCTCAAACC- ${ }^{\prime}$ and reverse: $5^{\prime}$-TCGAAGCTGTTGTCTTAACTCTGAC-3'), Tagln (forward: $\quad 5^{\prime}$-CTCTAATGGCTTTGGGCAGTTTG-3' and reverse: $5^{\prime}$-TGCAGTTGGCTGTCTGTGAAGTC-3'), Myhl1 (forward: 5'-GACAACTCCTCTCGCTTTGG- $3^{\prime}$ and reverse: 5'-GCTCTCCAAAAGCAGGTCAC-3'), and Vcaml (forward: $5^{\prime}$-AGTTGGGGATTCGGTTGTTCT-3' and reverse: 5'-CCCCTCATTCCTTACCACCC-3').

Unknown samples were run at the equivalent of $4 \mathrm{ng}$ of cDNA per well for each gene target (16 ng per well for Fap).
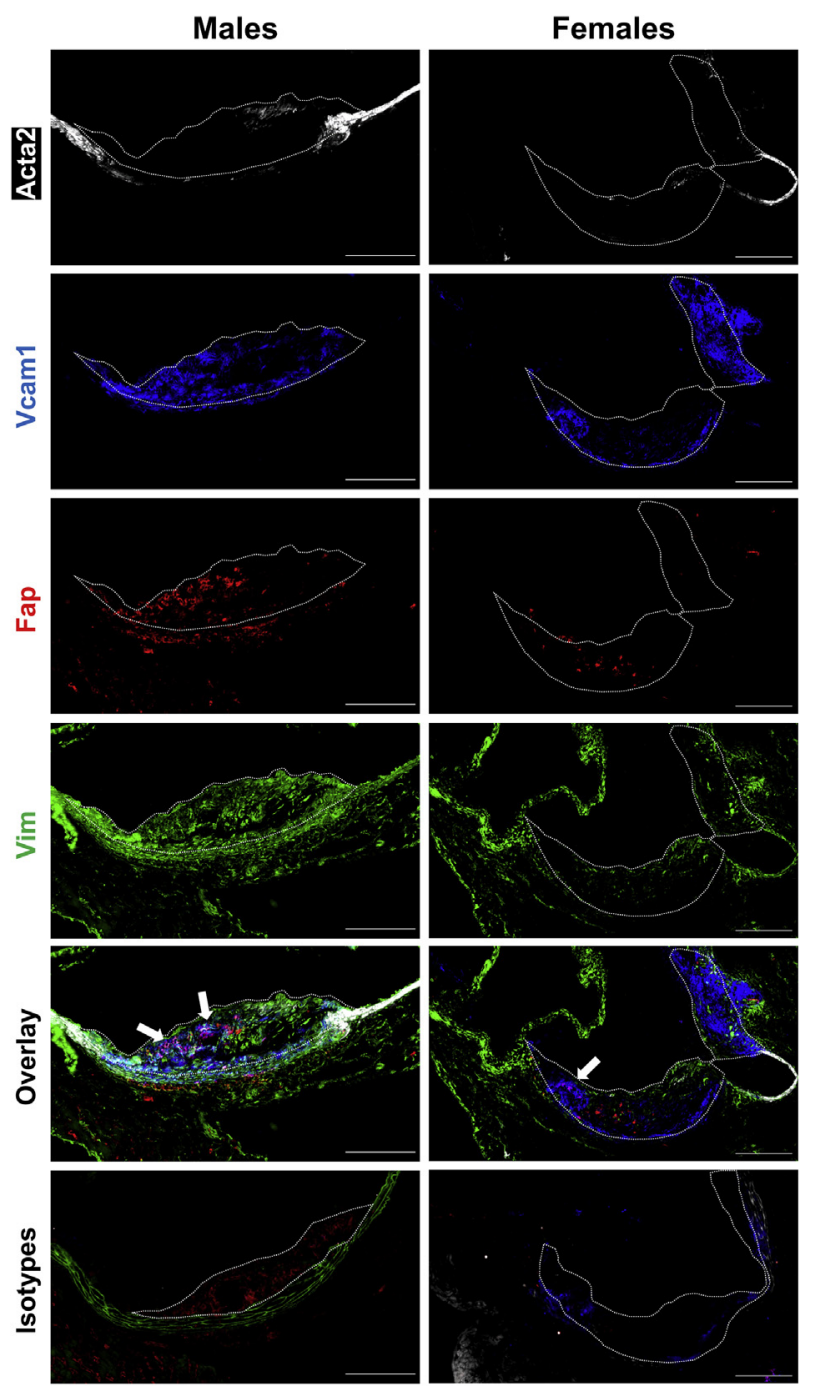

Figure 3 Fibroblast activation protein (Fap) expression defines a subpopulation of mesenchymal cells. Male and female $\mathrm{Apoe}^{-/-}$mice were maintained on a chow diet and aged to 26 weeks. Multiplex staining of $10-$ $\mu \mathrm{mol} / \mathrm{L}$ frozen sections of aortic roots was performed with antibodies targeted to mesenchymal vascular smooth muscle cells markers $\alpha$-smooth muscle actin (Acta2) (white), vascular cell adhesion molecule 1 (Vcam1) (blue), Fap (red), and vimentin (Vim) (green). Atherosclerotic lesions are highlighted with dotted lines. Arrows highlight Fap/Vcam1 overlapping regions (magenta). Scale bars $=200 \mu \mathrm{m}$.

Reactions were run on a StepOne Plus Real-Time PCR System (Applied Biosystems). Mean expression levels of gene targets were calculated from standard curves using StepOne Plus software version 3.0 (Applied Biosystems), normalized to the housekeeping gene (Gapdh), and graphed.

\section{Digital Imaging and Quantification}

Digital images were captured on a Nikon TiE inverted microscope (Nikon Instruments Inc., Melville, NY). The motorized stage and automated image stitching were used to obtain complete images of each aortic root. Histochemical and immunohistochemical stains were captured using brightfield 

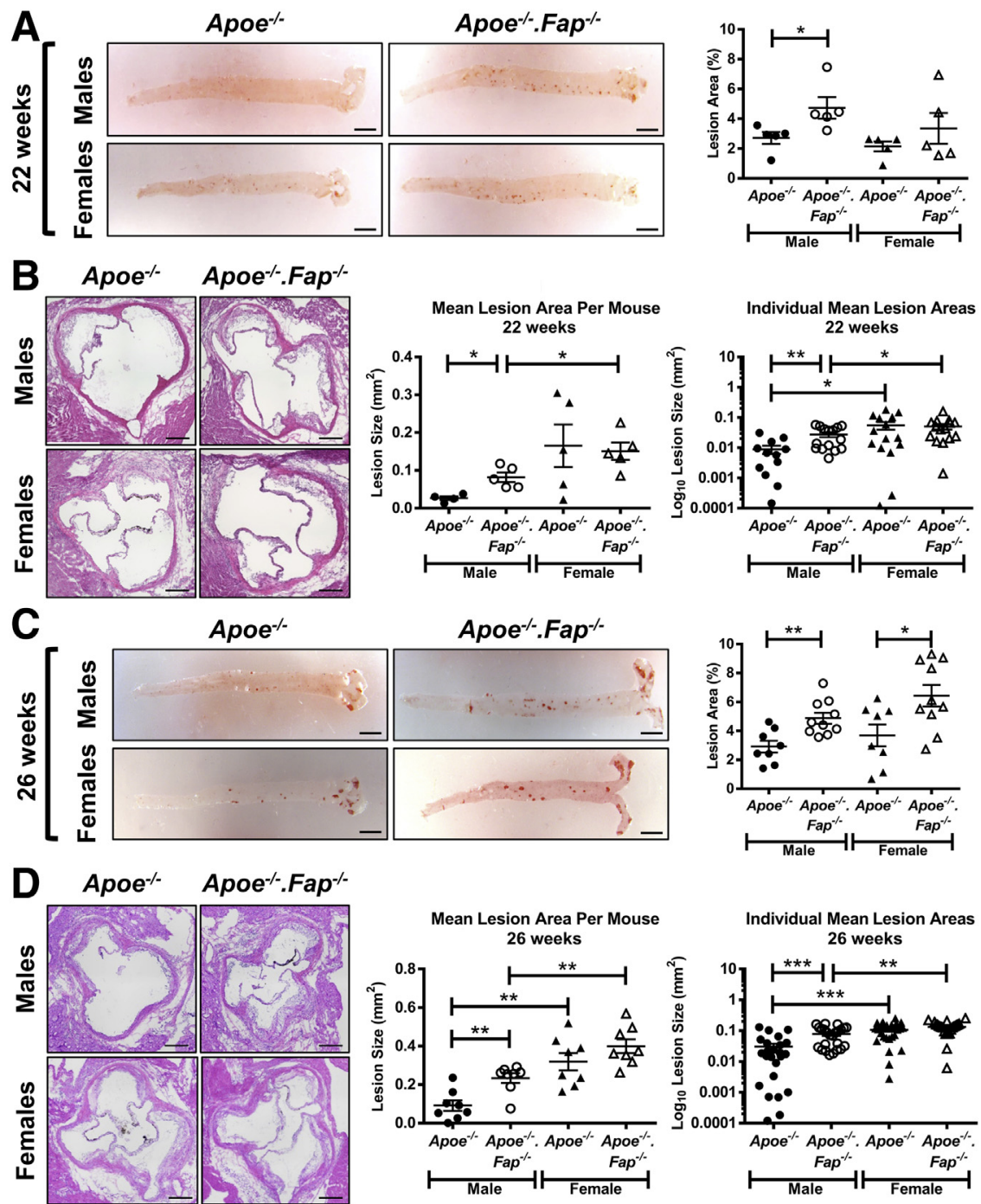

Figure 4 Deletion of fibroblast activation protein (Fap) accelerates atherosclerotic lesion progression in vivo. Male and female $\mathrm{Apoe}^{-/-}$and Apoe $^{-1-}$. Fap $^{-1-}$ mice were maintained on a chow diet and aged to 22 or 26 weeks. A and C: Aortas were isolated and stained ex vivo with 0il Red 0 to visualize atherosclerotic lesions (red). Lesion burden was quantified as percentage of lesion area of the total aortic area. B and D: $10-\mu \mathrm{mol} / \mathrm{L}$ frozen cross-sections of aortic roots were stained with hematoxylin and eosin to visualize lesions. Lesion area was quantified and graphed as separate data points for each lesion or as mean lesion area per mouse. $n=4$ to 5 mice (12 to 15 aortic root lesions) at 22 weeks and 8 to 10 mice (24 aortic root lesions) at 26 weeks. ${ }^{*} P \leq 0.05,{ }^{*} P \leq 0.01$, and $* * * P \leq 0.001$. Scale bars: $2.5 \mathrm{~mm}(\mathbf{A}$ and $\mathbf{C}) ; 200$ $\mu \mathrm{m}$ (B and $\mathbf{D})$. optics and a Nikon DSRi 2 color camera at $\times 10$ magnification. Picrosirius Red-stained samples were imaged using circularpolarized light optics to view fibrillar collagen and captured on a CCD Hammamatsu Camera at $\times 20$ magnification. Multichannel immunofluorescent samples were captured on the CCD Hammamatsu Camera at $\times 20$ magnification, using independently controlled excitation and emission wavelength filter wheels for each fluorophore. NIS Elements software version 4.60.00 (Nikon Instruments) was used for image quantification and pseudo-coloring of immunofluorescent images, where appropriate. For calculation of aortic root lesion burden (from hematoxylin and eosin images), individual lesion areas across the six stained sections were averaged. Lesion content for all other markers was calculated by thresholding the positive area and calculating a percentage of the total lesion area. The co-localization area to determine ECM compartments and macrophage subtype content was calculated from the appropriate overlapping thresholded immunofluorescent channels using NIS Elements software.

\section{Statistical Analysis}

All results were graphed as means \pm SEM. Statistical analysis between comparable pairs of groups or multiple groups in vivo was performed using the $t$-test or two-way analysis of variance test, respectively. Multiple-grouped analysis for VSMC phenotype in vitro was performed using the $\chi^{2}$ test. All statistical tests were calculated using Prism software version 6.0 (GraphPad Software, La Jolla, CA). $P \leq 0.05$ was considered to be statistically significant.

\section{Results}

Kinetics and Localization of Fap Expression to VSMCRich Regions but Not Inflammatory Cell-Rich Regions

To determine the spatial and temporal distribution of Fap expression in atherosclerosis, male and female $A_{p o e^{-/-}}$ mice that spontaneously develop disease were maintained 

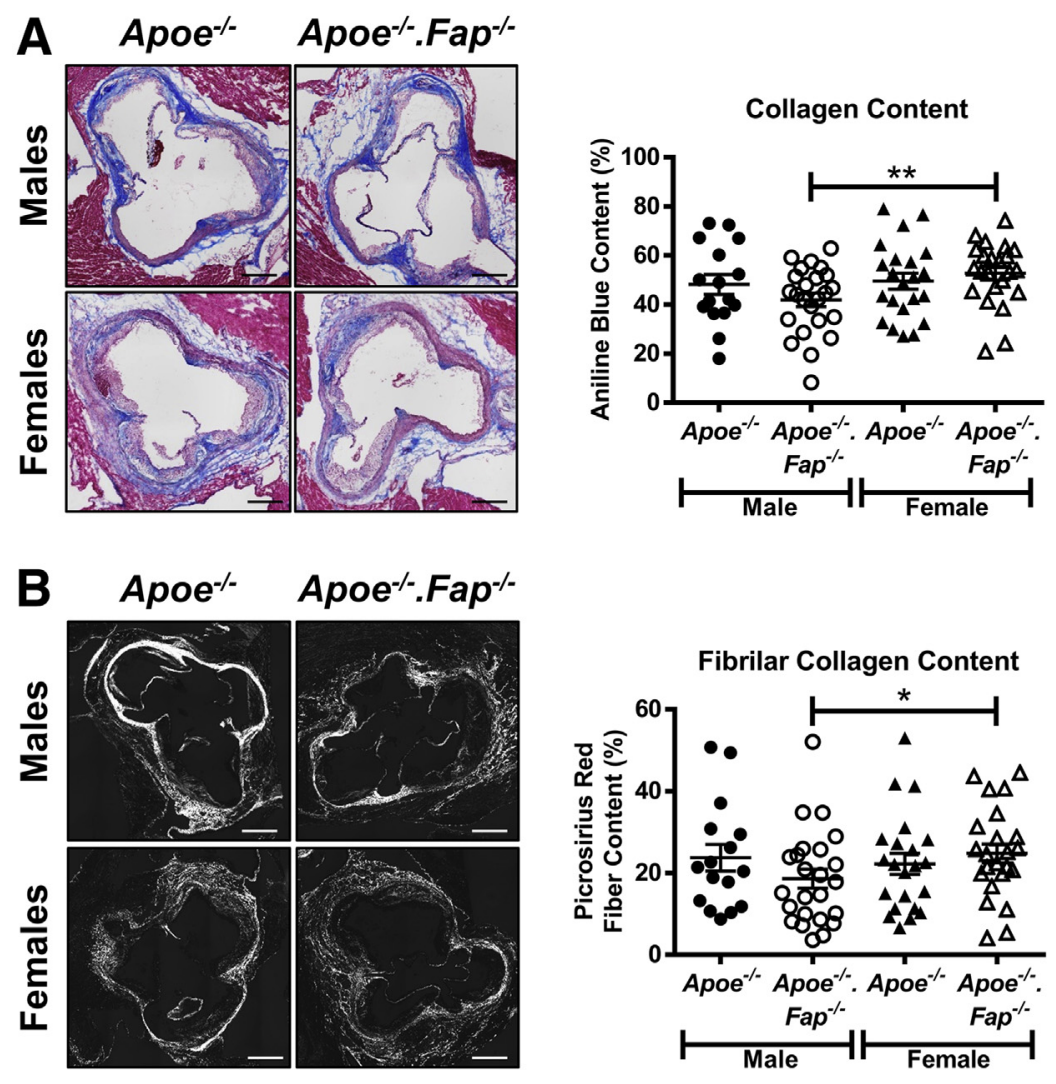

Figure 5 Sexually dimorphic effect of fibroblast activation protein (Fap) deletion on atherosclerotic lesion lipid and collagen content. Male and female $\mathrm{Apoe}^{-/-}$and $\mathrm{Apoe}^{-/-}$. $\mathrm{Fap}^{-/-}$mice were maintained on a chow diet and aged to 26 weeks. Then $10-\mu \mathrm{m}$ frozen cross-sections of aortic roots were stained with modified Masson's Trichrome (total collagen) (A), Picrosirius Red (fibrillar collagen when observed through circular polarized light) (B), and Oil Red 0 (lipids) (C). Total collagen, fibrillar collagen, and lipid content were then quantified and graphed as the percentage of the total area per lesion. ${ }^{*} P \leq 0.05,{ }^{* *} P \leq 0.01$, and ${ }^{* * *} P \leq 0.001$. Scale bars $=200 \mu \mathrm{m}$.
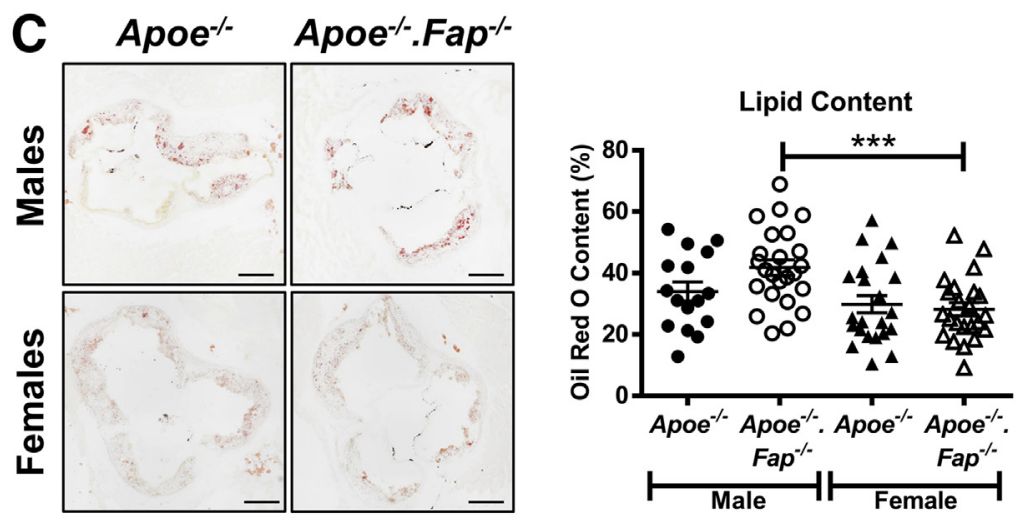

on a normal chow diet. Mice were euthanized at 22, 26, 30, or 34 weeks of age, and OCT-embedded frozen sections of the aortic roots were immunostained for Fap (Figure 1) or an IgG isotype control (Supplemental Figure S1). Fap was barely detectable in early atherosclerotic lesions in males at 22 weeks, although it was detected in lesions in females at the same time point. Fap was commonly observed in lesions in both sexes at 26, 30, and 34 weeks of age. In males, Fap expression followed a temporal normal distribution during these time points, with the highest levels detected at 30 weeks (Supplemental Figure S2A). Interestingly, Fap expression in female Apoe $e^{-/-}$mice did not follow a similar pattern, instead displaying comparable Fap expression during each of the time points analyzed (Supplemental
Figure S2A). Furthermore, lesion size in female mice was comparable across all time points and more than likely explains why Fap expression was observed more rapidly in female mice compared with male mice and sustained at late time points. Going forward, the 26-week-old time point was chosen to further study Fap because this was the earliest time point in both sexes that displayed robust and comparable Fap expression.

To broadly characterize the localization of Fap in lesions, aortic root sections from 26-week-old male and female Apoe ${ }^{-1-}$ mice were co-immunostained for Fap and broadspectrum markers of mesenchymal (Vim) and inflammatory cells (Cd68) (Figure 2). Vim and Cd68 were used because they clearly identified nonoverlapping mesenchymal and 


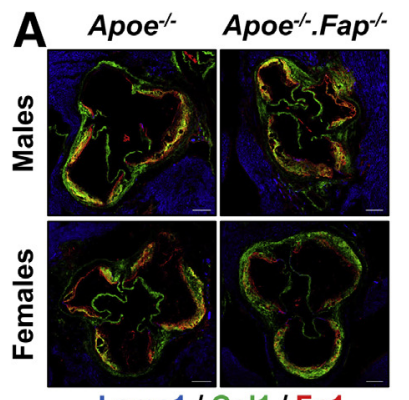

Lama1 / Col1 / Fn1
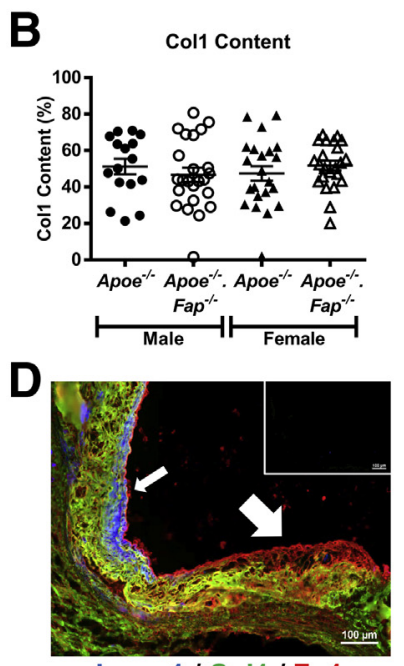

Lama1 / Col1 / Fn1

$\mathbf{F}$

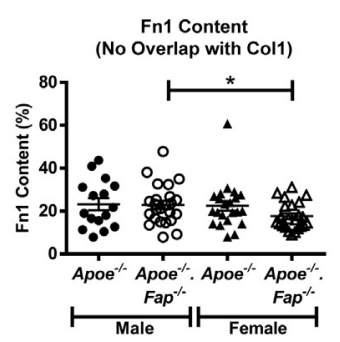

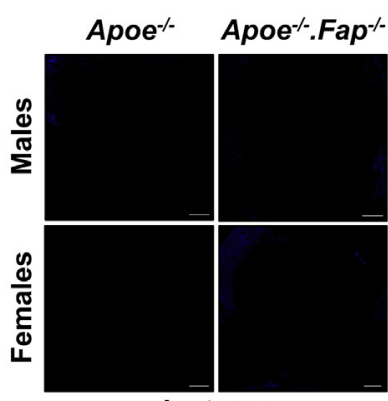

Isotypes

E

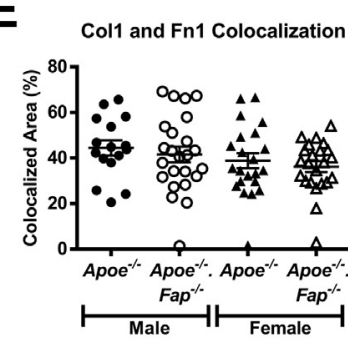

G

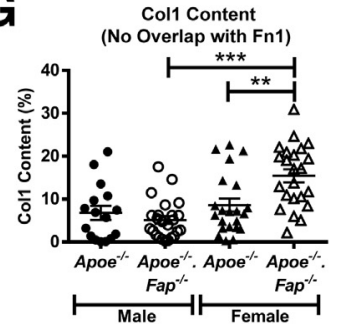

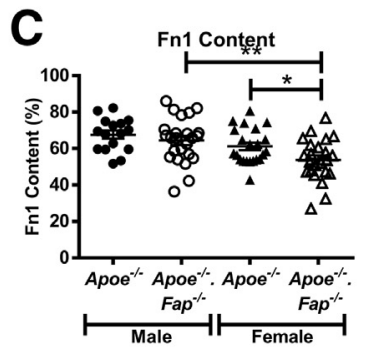

Figure 6 Increase in collagen type I (Col1)-rich and concomitant reduction in fibronectin (Fn1)-rich regions of lesions in female fibroblast activation protein (Fap) -deficient mice. A: $10-\mu \mathrm{m}$ frozen crosssections of aortic roots from 26 -week-old male and female $A p o e^{-/-}$ and $\mathrm{Apoe}^{-/-}$. $\mathrm{Fap}^{-/-}$mice were stained with antibodies targeted to Lama1 (blue), Col1 (green), and Fn1 (red) or isotype IgG controls (A). Total lesional Col1 (B) and Fn1 (C) content was quantified and graphed. D: Higher magnification of lesions shows that Lama1 localizes only to cap regions (small arrow), whereas Col1 and Fn1 stain separate compartments (large arrow). Inset: IgG isotype controls. Areas of colocalized Col1 and Fn1 regions (E) as well as Fn1-only (F) and Col1-only (G) regions were also graphed. ${ }^{*} P \leq 0.05,{ }^{* *} P \leq 0.01$, and $* * * P \leq 0.001$. Scale bars: $200 \mu \mathrm{m}($ A) $; 100 \mu \mathrm{m}$ (D, main image). Original magnification, $\times 20$ (D, inset)

inflammatory regions, respectively (Figure 2). Fap staining showed little to no overlap with Cd68, whereas positive costained regions (white arrows highlighting yellow areas) for Fap and Vim were commonly observed (Figure 2). This pattern of Fap co-localization was observed in both sexes. These data indicate that Fap expression in atherosclerotic lesions was limited to mesenchymal cell-rich areas as previously reported in human plaques ${ }^{34}$ and not detectably expressed on inflammatory cells within atherosclerotic lesions.

\section{Fap Expression Defines a Subpopulation of Lesional Dedifferentiated Mesenchymal Cells in Atherosclerosis}

Lesional mesenchymal cells, of which most are of VSMC origin, represent a heterogeneous population in atherosclerosis. Recent lineage tracing studies have cemented the plasticity of VSMCS and revealed that a high percentage of cells with a VSMC origin can take on markers of inflammatory/macrophage-like cells, including Cd68 and LGALS3. ${ }^{11,41,42}$ The above data suggested that Fap was not expressed on $\mathrm{Cd}_{68}{ }^{+}$cells irrespective of their origin. However, there is as yet no clear single marker for noninflammatory (transitioned) lesional mesenchymal cells. To better determine if Fap was expressed in populations of nontransitioned mesenchymal cells in lesions, multiplex immunostaining for Fap was performed with multiple previously characterized markers of lesional mesenchymal cells (Acta2, Vcam1, and Vim) on aortic root sections from 26-week-old Apoe ${ }^{-/-}$mice (Figure 3). ${ }^{11,35,37,41-47}$ These markers spatially and generally characterize mesenchymal cells into two populations: lesional dedifferentiated mesenchymal cells in the core of lesions (Vcam1) versus lesional fibrotic mesenchymal cells that have undergone fibrotic redifferentiation in the fibrotic cap (Acta2). In both males and females, Acta2 expression was limited to medial VSMCs located in non-lesion-bearing vessel walls on the lesion periphery as well as in lesional fibrotic mesenchymal cells in early partial fibrotic cap formations. Vcam1 was limited to neointimal regions and was nonoverlapping with Acta2, confirming the presence of previously characterized lesional dedifferentiated mesenchymal cells. ${ }^{35,37,43-47}$ Fap expression was predominantly localized to lesions and occasionally to adventitia. Adventitial Fap expression co-localized with Vim but not Acta2, suggesting that these cells were a population of activated fibroblasts, as described elsewhere. ${ }^{48}$ Lesional Fap expression showed no overlap with Acta2, indicating that Fap was not expressed at detectable levels by either medial differentiated VSMCs in the vessel wall or lesional fibrotic mesenchymal cells in fibrotic caps. Lesional Fap expression frequently co-localized with Vcam1 (white arrows highlighting magenta color), suggesting that Fap was expressed on lesional dedifferentiated mesenchymal cells.

Interestingly, there were instances where Fap was detected in lesions in the absence of Vcam 1 and vice versa (Figure 3). These areas were also positive for Vim, suggesting that Fap expression in the absence of $\mathrm{Vcam} 1 \mathrm{Fap}^{+}$, $\mathrm{Vcam}^{-}$) or vice versa $\left(\mathrm{Fap}^{-}, \mathrm{Vcam}^{+}\right)$were distinguishing subpopulations of lesional dedifferentiated mesenchymal cells. This study also aimed to determine the temporal expression of Vcam1 and Acta2 in lesions relative to Fap, which may help determine the fibrotic state of the disease (Supplemental Figure S2, B-D). Vcam1 expression 

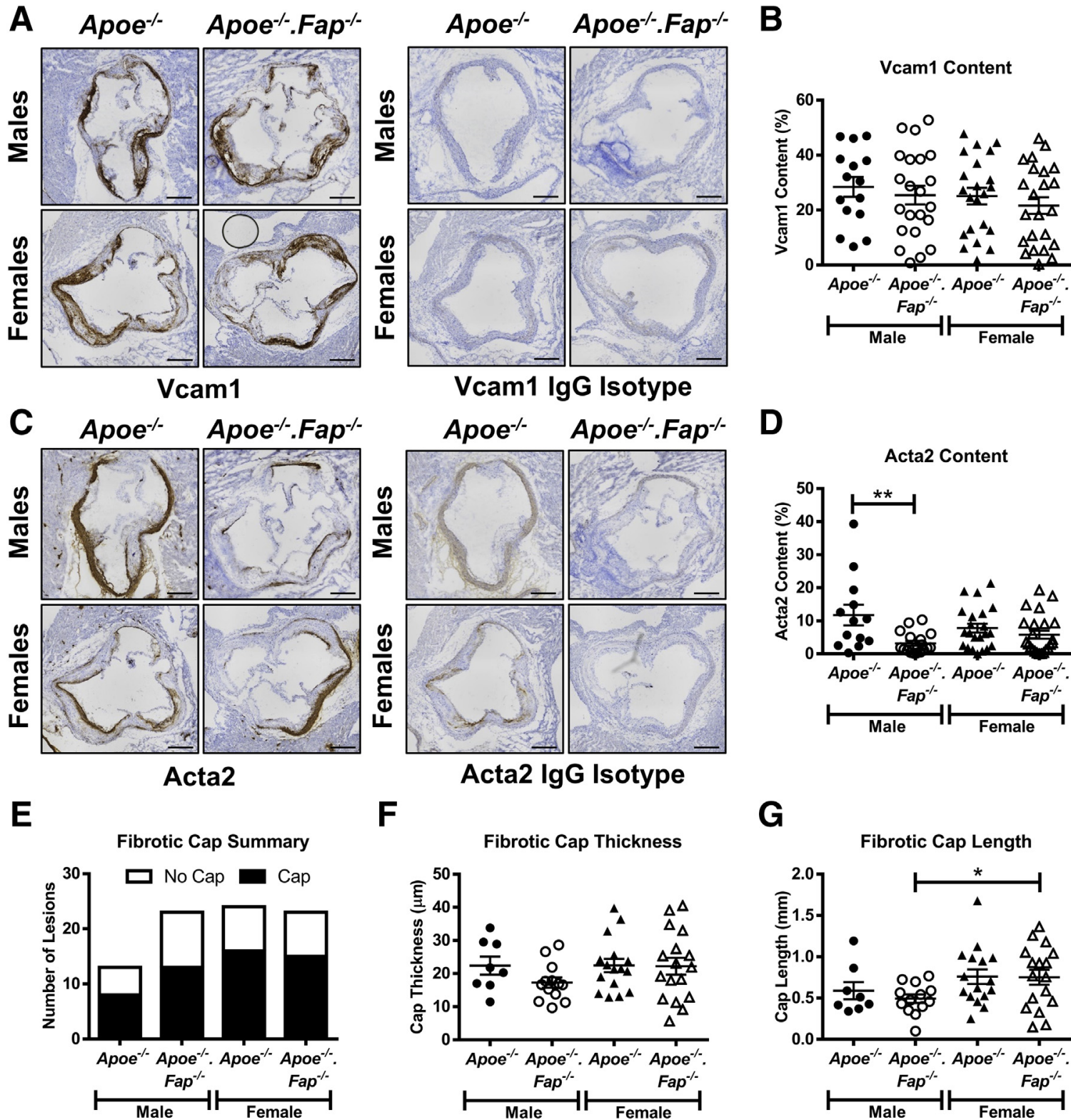

G

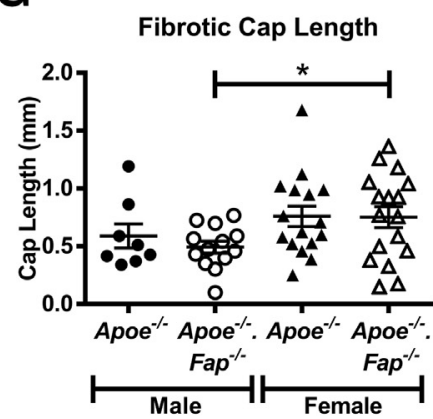

Figure 7 Fibroblast activation protein (Fap) deletion does not alter vascular smooth muscle cell (VSMC) phenotype in lesions. Frozen cross-sections of aortic roots $10 \mu \mathrm{m}$ thick from 26-week-old male and female $\mathrm{Apoe}^{-/-}$and $\mathrm{Apoe}^{-/-}$. Fap ${ }^{-{ }^{-}}$mice were stained with antibodies targeted to vascular cell adhesion molecule 1 (Vcam1) or IgG isotype control (A) and $\alpha$-smooth muscle actin (Acta2) or IgG isotype control (C). Lesional Vcam1 (B) and Acta2 (D) content was quantified and graphed. Lesions were also analyzed for the absence or presence of fibrotic caps $(\mathbf{E})$, cap thickness (F), and cap length $(\mathbf{G})$. ${ }^{*} P \leq 0.05,{ }^{*} P \leq 0.01$. Scale bars $=200 \mu \mathrm{m}$.

displayed a similar temporal expression pattern to that observed for Fap (Supplemental Figure S2B), whereas Acta2 expression did not have any significant differences in expression among the three time points analyzed (Supplemental Figure S2C). Regression analysis of Fap versus Acta2 expression (Supplemental Figure S2D) had no significant correlation, suggesting that the number of Fap ${ }^{+}$ lesional dedifferentiated mesenchymal cells is not dependent on and cannot predict the level of $\mathrm{Acta}^{+}$lesional fibrotic mesenchymal cells.

To further explore if FAP was specifically expressed on VSMCs within this mesenchymal compartment, cultured primary VSMCs isolated from thoracic aortas of wild-type C57BL/6 mice were used (Supplemental Figure S3). When plated on tissue culture plastic in media that contained $10 \%$ fetal bovine serum, primary VSMCs conformed to a heterogenous population that included a diversity of cell shapes and varying levels of Vcam1 and Acta2 protein expression consistent with the heterogeneity of mesenchymal cells observed in atherosclerotic lesions in vivo (Supplemental Figure S3, A and B). It has been reported that TGF- $\beta 1$ regulates VSMC phenotype and function in $\mathrm{CVD}^{49}$ and is thought to promote the generation of $\mathrm{Acta}^{+}$lesional fibrotic VSMCs as found in fibrotic caps in vivo. Indeed, 


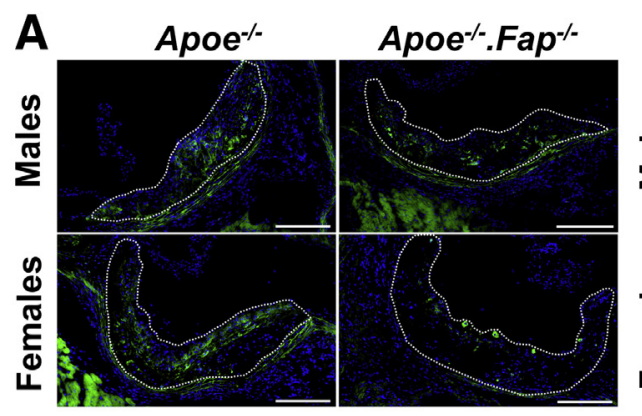

DAPI / Adgre1

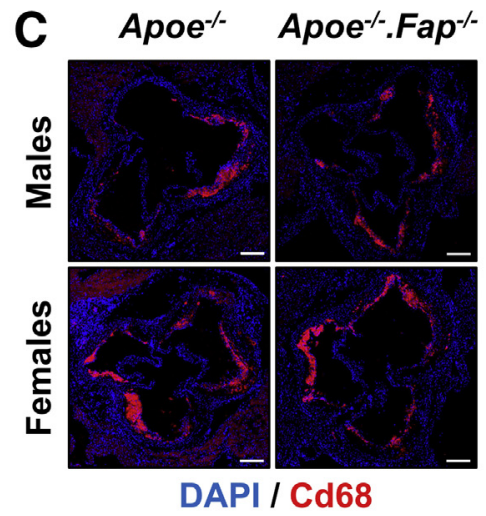

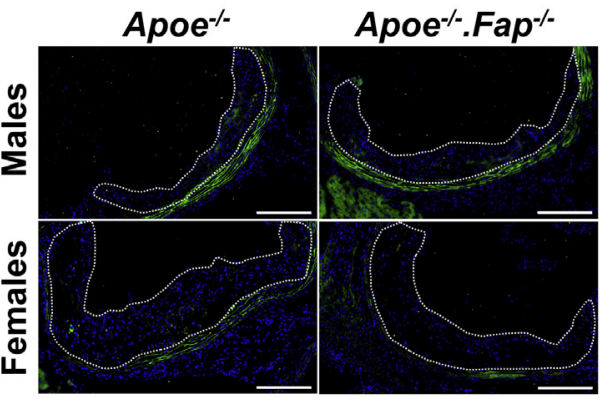

DAPI / IgG Isotype
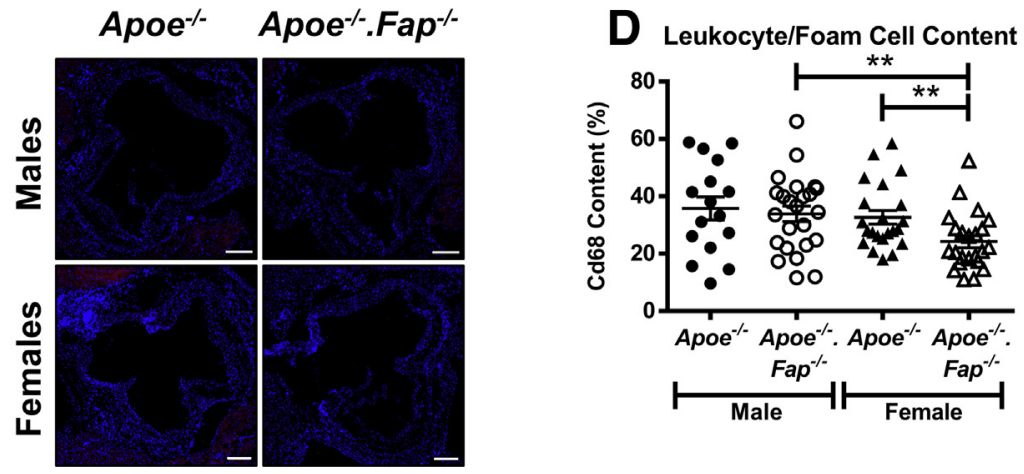

DAPI / IgG Isotype

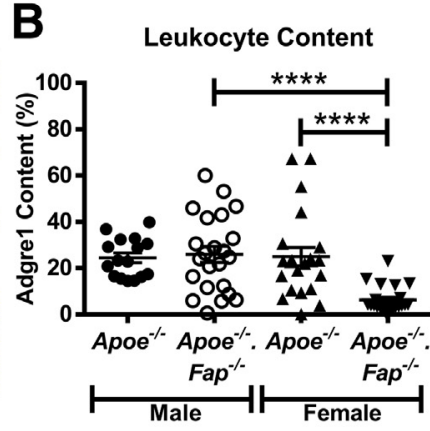

Figure 8 Reduced inflammatory cell content of lesions in fibroblast activation protein (Fap)-deficient female mice. Frozen cross-sections of aortic roots $10 \mu \mathrm{m}$ thick from 26-week-old male and female $A p o e^{-/-}$and $A p o e^{-/-}$. Fap ${ }^{-/-}$mice were stained with antibodies targeted to adhesion $\mathrm{G}$ protein-coupled receptor E1 (Adgre1) (green) or IgG isotype control (A) and Cd68 (red) or IgG isotype control (C). Lesional Adgre1 ${ }^{+}$monocyte/macrophage (B) and Cd68 ${ }^{+}$ macrophage/foam cell (D) content were quantified and graphed. Fluorescent signal observed in $\mathbf{A}$ outside the lesions is tissue autofluorescence that resulted from elastin in the vessel wall or cardiomyocytes in the surrounding myocardium. Atherosclerotic lesions are highlighted with dotted lines. ${ }^{\star} P \leq 0.01$, $\star * * * P \leq 0.0001$. Scale bars $=200 \mu \mathrm{m}$.

treatment with $2 \mathrm{ng} / \mathrm{mL}$ of TGF- $\beta 1$ caused a transition to a more uniform population of spindle-shaped cells, a significant increase in Acta2 expression, and a concomitant decrease in Vcam1 expression, a phenotype reminiscent of the lesional fibrotic mesenchymal cells observed in vivo (Supplemental Figure S3, A and B). Quantitative PCR analysis confirmed that TGF- $\beta 1$ promoted a fibrotic VSMC gene expression profile, including increased expression of Acta2, Tgln, and Myocd (Supplemental Figure S3C). Furthermore, this finding was coupled with a significant decrease in the expression of genes associated with lesional dedifferentiated mesenchymal cells, including Vcaml and Fap (Supplemental Figure S3D). These data further supported the notion that Fap is selectively expressed on lesional dedifferentiated VSMCs relative to medial differentiated VSMCs or lesional fibrotic VSMCs and suggests that TGF- $\beta 1$ may contribute to the generation of the lesional fibrotic VSMC phenotype.

\section{Deletion of Fap Increases Atherosclerotic Lesion Progression in Vivo}

The above data confirmed the expression of Fap in the Apoe $^{-/-}$murine model of atherosclerosis. To determine the net effect of Fap on the progression of the disease, Apoe $e^{-/-}$ mice were crossed to $\mathrm{Fap}^{-1-}$ mice to generate genetically engineered $\mathrm{Apoe}^{-/-} . \mathrm{Fap}^{-/-}$mice. Male and female Apoe $\mathrm{e}^{-/-}$ and $\mathrm{Apoe}^{-/-}$. $\mathrm{Fap}^{-1-}$ mice fed a chow diet were aged to 22 or 26 weeks and then compared for atherosclerotic lesion burden (Figure 4). At 22 weeks of age, aortic Oil Red O staining showed a significant increase in atherosclerotic lesion area in male $\mathrm{Apoe}^{-/-}$.Fap $^{-/-}$mice compared with Apoe $^{-/-}$mice. No differences in lesion area were observed in female Apoe $^{-/-} . \mathrm{Fap}^{-/-}$mice compared with $\mathrm{Apoe}^{-/-}$ mice at this time point (Figure 4A). Cross-sectional lesion area analysis of hematoxylin and eosin-stained aortic root sections also showed a significant increase in lesion area (either per individual lesion or mean lesion area per mouse) in male Apoe ${ }^{-1-}$. Fap ${ }^{-1-}$ mice compared with Apoe ${ }^{-/-}$mice (Figure 4B), but there was no difference between the two genotypes among female mice at 22 weeks of age (Figure 4B). At 26 weeks of age, lesion burden continued to be significantly accelerated in male $\mathrm{Apoe}^{-/-}$. Fap $^{-/-}$mice compared with $A p o e^{-1-}$ mice, as shown by Oil Red O staining of aortas as well as in the aortic root, respectively (Figure 4, C and D). Moreover, by 26 weeks of age lesion burden in aortas of female Apoe . Fap $^{-1-}$ mice was also significantly increased over Apoe ${ }^{-/-}$(Figure 4C), although 

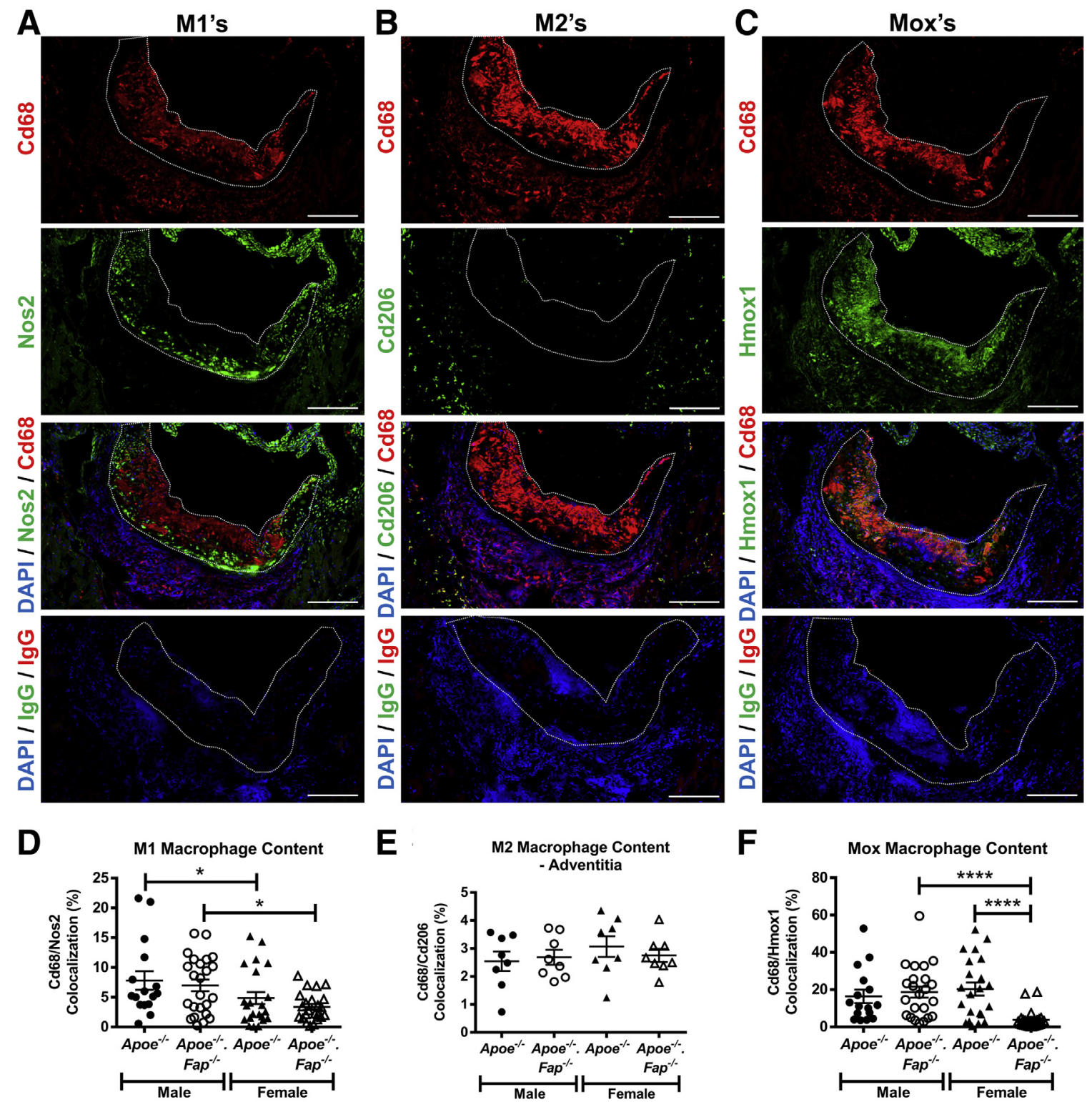

Figure 9 Reduced accumulation of M1 and Mox macrophages in lesions of fibroblast activation protein (Fap)-deficient female mice. Frozen cross-sections of aortic roots $10 \mu \mathrm{m}$ thick from 26-week-old male and female $\mathrm{Apoe}^{-/-}$and $\mathrm{Apoe}^{-/-}$. Fap ${ }^{-{ }^{-}}$mice were co-immunostained with antibodies targeted for $\mathrm{Cd} 68$ (red) and Nos2 (green) for M1s (A), Cd68 (red) and Cd206 (green) for M2s (B), and Cd68 (red) and heme oxygenase (Hmox1) (green) for Moxs (C). Atherosclerotic lesions are highlighted with dotted lines. M1 (D), M2 (E), and Mox (F) content was quantified and graphed. ${ }^{*} P \leq 0.05, * * * * P \leq 0.0001$. Scale bars $=200 \mu \mathrm{m}$.

there was still no difference in lesion burden in crosssections of their corresponding aortic roots at this time point (Figure 4D). Consistent with a prior study using Apoe $^{-/-}$mice ${ }^{50}$ aortic root lesions in female $\mathrm{Apoe}^{-/-}$and Apoe $^{-1-} . \mathrm{Fap}^{-1-}$ mice were consistently significantly larger than their genetic counterparts in male mice at both 22 and 26 weeks of age (Figure 4, B and D). The differences in lesion burden between genotypes were independent of changes in circulating levels of serum cholesterol or triglycerides, which were comparable between genotypes (Supplemental Figure S4). Thus, Fap restrains atherosclerosis in both sexes, but the effect is detectable earlier in males.
Sexually Dimorphic Impact of Fap Deletion of Matrix and Lipid Content of Atherosclerotic Lesions

We sought to determine if the increase in atherosclerotic lesion burden in Fap-deficient mice coincided with morphologic changes within lesions. For accurate comparison, all subsequent analyses of lesion content described below were based on comparisons between similar-sized aortic root lesions with an area of $\log _{10} \geq 0.01 \mathrm{~mm}^{2}$ (Figure 4D). The proteolytic activity of Fap is known to be intricately involved in the ordered enzymatic degradation of fibrillar collagen that affects cellular behavior and matrix 
Table 2 Summary of Profiling of Atherosclerotic Lesion Composition

\begin{tabular}{|c|c|c|c|c|}
\hline \multirow[b]{2}{*}{ Lesion feature } & \multicolumn{2}{|l|}{ Males } & \multicolumn{2}{|l|}{ Females } \\
\hline & $a p o E^{-/-}$ & $a p o E^{-/-} . F A P^{-/-}$ & $a p o E^{-/-}$ & $a p o E^{-/-} . F A P^{-/-}$ \\
\hline Aortic lesion burden & + & ++ & + & ++ \\
\hline Lipid content & + & + & + & + \\
\hline Total collagen & + & + & + & + \\
\hline Fibrillar collagen & + & + & + & + \\
\hline Col1/Fn1 colocalization & + & + & + & + \\
\hline Col1 only (no overlap with Fn1) & ++ & + & ++ & +++ \\
\hline Fn1 only (no overlap with Col1) & ++ & ++ & ++ & + \\
\hline Vcam1 & + & + & + & + \\
\hline Acta2 & ++ & + & ++ & ++ \\
\hline $\mathrm{Cd} 68^{+}$inflammatory cells & ++ & ++ & ++ & + \\
\hline M1 macrophages & ++ & ++ & + & + \\
\hline M2 macrophages & + & + & + & + \\
\hline Mox macrophages & ++ & ++ & ++ & + \\
\hline
\end{tabular}

Lesion feature detected at moderate $(+)$, increased $(++)$, and highly abundant $(+++)$ levels.

Acta2, $\alpha$-smooth muscle actin; Adgre1, adhesion G protein-coupled receptor E1; Col1, collagen type I; Fn1, fibronectin; Vcam1, vascular cell adhesion molecule 1.

content in other disease settings. ${ }^{17-19,24-32,51}$ Male and female Apoe $^{-/-}$and Apoe ${ }^{-1-}$.Fap ${ }^{-/-}$mice 26 weeks old were therefore first analyzed for total collagen, fibrillar collagen, and lipid content by staining aortic root cross-sections with a modified Masson's Trichrome, Picrosirius Red, and Oil Red O, respectively (Figure 5). Genetic deletion of Fap did not alter total collagen (Figure 5A), fibrillar collagen (Figure 5B), or lipid content (Figure 5C) in male or female Apoe ${ }^{-/-}$mice. Moreover, no significant differences were found in any of these parameters between lesions in male $\mathrm{Apoe}^{-/-}$versus female $\mathrm{Apoe}^{-/-}$mice. Interestingly, however, lesions in female $\mathrm{Apoe}^{-1-} . \mathrm{Fap}^{-1-}$ mice exhibited increases in total and fibrillar collagen content as well as decreased lipid content compared with male Apoe $^{-/-}$. Fap ${ }^{-/-}$counterparts (Figure 5 and Supplemental Figure S5A). Thus, deletion of Fap revealed a sex-specific difference in the fibrotic component of Apoe ${ }^{-/-}$lesions.

This study further compared matrix protein content of lesions by multiplex immunostaining of aortic root sections of Apoe ${ }^{-/-}$and Apoe $e^{-/}$.Fap ${ }^{-/-}$mice for Col1, Fn1, and Lama1 (Figure 6). Lamal staining was abundant in the surrounding cardiac muscle but was minimal within neointima of lesions. Lama1 was, however, detected in fibrotic caps when present (Figure 6, A and E). In contrast, Coll and Fn1 were abundant in lesions (Figure 6A). No changes were observed in lesional Coll area between groups (Figure 6B), although a modest yet significant decrease in the mean intensity of the Col1 (Supplemental Figure S5B) was observed in male Apoe ${ }^{-1-}$ Fap $^{-/-}$mice compared with male $A p o e^{-/-}$mice. In addition, female Apoe $^{-/-}$. Fap $^{-1-}$ mice had increased Col1 signal intensity compared with male $\mathrm{Apoe}^{-/-} . \mathrm{Fap}^{-/-}$mice consistent with data for total collagen presented in Figure 5 (Supplemental Figure S5B). Fn1 lesional area or staining intensity was unchanged in male $\mathrm{Apoe}^{-/-}$. Fap ${ }^{-1-}$ mice compared with male Apoe $e^{-/-}$ mice (Figure 6C and Supplemental Figure S5C). However, female $\mathrm{Apoe}^{-1-} . \mathrm{Fap}^{-1-}$ mice had a significantly decreased Fn1 area compared with female Apoe $e^{-/}$mice and compared with male Apoe ${ }^{-/-} . \mathrm{Fap}^{-/-}$mice (Figure 6C). Thus, the sexually dimorphic effect of Fap deletion on matrix content was evident based on the effect on both Col1 and Fn1. No changes were observed in total Fn1 staining intensity between the female groups (Supplemental Figure S5C).

Interestingly, analysis of the distribution of immunostaining clearly demonstrated that Col1 and Fn1 were localized to distinct regions within lesions (Figure 6D). Specifically, $\mathrm{Fn}^{+}$areas (nonoverlapping with Col1) were commonly found on the luminal side of the neointima (large arrow), whereas Fn1-Col1 overlapping regions and Col1 ${ }^{+}$ areas (nonoverlapping with $\mathrm{Fn} 1$ ) were localized to the central areas of lesions and more proximal to the media, respectively. We hypothesized that regional heterogeneity in matrix composition may reflect regional heterogeneity of distinct cellular phenotypes and therefore investigated whether this regional heterogeneity was altered on global deletion of Fap. Fn1-Col1-I double-positive areas were unchanged between genotypes or based on the sex of the mice (Figure 6E). Col1-I signal intensity in this subregion was decreased in male Apoe $e^{-/-}$. Fap $^{-/-}$mice compared with 
male $\mathrm{Apoe}^{-/-}$mice and female $\mathrm{Apoe}^{-/-} \cdot \mathrm{Fap}^{-/-}$mice (Supplemental Figure S5D). Fn1 staining intensity in this subregion remained unchanged between groups (Supplemental Figure S5E). Fn $1^{+}$subregions (nonoverlapping with Col1) were not different in area or signal intensity between genotypes within the male and female cohorts (Figure 6F and Supplemental Figure S5F). However, Fn1 staining intensity in this subregion was modestly yet significantly increased in female $\mathrm{Apoe}^{-/-} . \mathrm{Fap}^{-/-}$mice compared with male Apoe ${ }^{-/-}$.Fap ${ }^{-/-}$mice (Supplemental Figure $\mathrm{S} 5 \mathrm{~F}$ ) despite a modest decrease in collagen area (Figure 6F). Finally, $\mathrm{Col1}^{+}$areas (nonoverlapping with Fn1) were unchanged between genotypes in male mice (Figure 6G), although Col1 signal intensity in this subregion was decreased in male Apoe $^{-/-}$. Fap ${ }^{-/-}$mice compared with the male Apoe ${ }^{-/-}$mice (Supplemental Figure S5G). However, in this subregion in female mice, the Coll area was significantly increased in $A p o e^{-/-} \cdot$ Fap $^{-1-}$ mice compared with $A p o e^{-/-}$mice, with no change in Coll intensity between these groups (Figure 6G and Supplemental Figure S5G). In addition, Col1 area and intensity were higher in this subregion in female Apoe $^{-/-}$.Fap ${ }^{-/-}$mice than their male genetic counterparts.

Taken together, these data further indicate that Fap may regulate atherosclerotic lesion content in a sexually dimorphic manner. In males, Fap deletion accelerated the disease without affecting lesional ECM phenotype. Conversely in females, Fap deletion also accelerated atherosclerosis that was accompanied by ECM phenotypic changes. These data also indicate that matrix composition can be used to map spatial heterogeneity in lesion architecture beyond neointima and fibrotic caps to define ECM-based subregions of neointima.

\section{Deletion of Fap Does Not Alter Lesional Mesenchymal Cell Subpopulations}

Next, it was determined if the matrix changes coincided with alterations in the subpopulations of lesional mesenchymal cells, the primary cells in lesions responsible for matrix deposition. Aortic root cross-sections from 26week-old male and female Apoe $e^{-1-}$ and Apoe ${ }^{-1-}$. Fap $^{-1-}$ mice were therefore immunostained for the spatially distinct markers Vcam1 and Acta2 (Figure 7). Lesional Vcam1 content was similar between groups (Figure 7, A and B). Medial Vcam1 expression was also comparable between the groups (Supplemental Figure S5H). Lesional Acta2 content in male $\mathrm{Apoe}^{-1-}$. $\mathrm{Fp}^{-1-}$ mice was significantly decreased when compared with male Apoe $e^{-1}$ mice, although no differences were observed between genotypes in female mice (Figure 7, C and D). Given the fact that lesional Acta2 expression was restricted to fibrotic caps, these were analyzed further. It is still unclear how $\mathrm{Acta} 2^{+}$fibrotic caps form, be it direct outgrowth from medial differentiated $\mathrm{Acta}^{+}$VSMCs in the vessel wall or a partial redifferentiation of lesional dedifferentiated $\mathrm{Vcam} 1^{+}$mesenchymal cells. The study therefore sought to determine if Fap was implicated in the occurrence and/or size of fibrotic caps in vivo. The occurrence of lesions with fibrotic caps was not affected in either sex on global deletion of Fap (Figure 7E). Of those lesions in which fibrotic caps were present, no significant changes in cap thickness (Figure 7F) or cap length (Figure $7 \mathrm{G}$ ) between genotypes were observed, although a small yet significant increase in cap length was observed in female Apoe ${ }^{-1-}$. Fap $^{-/-}$mice when compared with male $\mathrm{Apoe}^{-1-}$.Fap $^{-1-}$ mice (Figure $7 \mathrm{G}$ ). These data together suggested that Fap did not significantly affect the relative prevalence or the regional distribution of mesenchymal cell subpopulations or fibrotic cap morphology.

\section{Sexually Dimorphic Fap-Dependent Regulation of Inflammation and Mox Macrophage Content in Atherosclerotic Lesions}

The above data have so far concerned how global deletion of Fap affected lesion morphology by analyzing the cellular and ECM components of the fibrotic response. It is well established that the recruitment and differentiation of circulating monocytes and lesional inflammation play just as important a role in atherosclerosis and that the balance between the inflammatory and fibrotic responses may be critical to disease outcome. To determine if Fap has the potential to also affect lesional inflammation, aortic root cross-sections from 26-week-old male and female $\mathrm{Apoe}^{-/-}$and $\mathrm{Apoe}^{-/-} \cdot \mathrm{Fap}^{-/-}$mice were immunostained for the pan-monocyte/macrophage marker F4/80 (Adgre1), macrophage/foam cell marker Cd68, or their respective isotype $\mathrm{IgG}$ controls (Figure 8). Adgre1 content was unchanged between genotypes in male mice but was significantly decreased in female $A p o e^{-/-}$.Fap $^{-/-}$mice compared with female $\mathrm{Apoe}^{-1-} . \mathrm{Fap}^{-1-}$ mice or male Apoe $\mathrm{C}^{-1-}$. Fap $^{-1-}$ mice (Figure 8, A and B). Lesional Cd68 content followed the same pattern, with significantly decreased Cd68 content observed in female $\mathrm{Apoe}^{-/-}$. $\mathrm{Fap}^{-/-}$mice compared with female Apoe $e^{-/-}$mice or male $a p o E^{-/-} . F A P^{-/-}$mice (Figure 8, $\mathrm{C}$ and $\mathrm{D}$ ). Combined, these data indicate that global deletion of Fap had a sexually dimorphic effect on lesional inflammation, with the effect greater in female than male mice.

Recent reports have suggested that different macrophage subpopulations, such as proinflammatory M1 macrophages, anti-inflammatory M2 macrophages, and oxidizing Mox macrophages can have counterbalancing roles in inflammation in the context of atherosclerosis. ${ }^{52}$ Therefore, the role of Fap in regulating the distribution of macrophage subpopulations in lesions was studied (Figure 9). Multiplex immunostaining for the pan macrophage marker Cd68 with specific markers for M1 (Nos2), M2 (Cd206), or Mox (Hmox1) macrophages showed striking spatial heterogeneity in the distribution of these various macrophage phenotypes. M1 macrophages were detected in lesions and occasionally in adventitia (Figure 9A). Contrarily, M2 macrophages were largely restricted to adventitia with only sparse representation within lesions (Figure 9B). Mox macrophages were abundant 
and accumulated in greater numbers than M1 macrophages within lesions (Figure 9C). Furthermore, female Apoe ${ }^{-/-}$ mice or female $a p o E^{-/-} . F A P^{-/-}$mice had reduced lesional M1 macrophage content than the respective genotypes in male mice, but no differences were observed between Fap genotypes within either sex (Figure 9D). No significant changes were detected between any groups in adventitial M2 macrophage content between Fap wild-type and Fapdeficient male or female mice (Figure 9E), although significant yet modest increases in the small numbers of M2 macrophages in lesions were observed in female $\mathrm{Apoe}^{-/-}$ mice or female $a p o E^{-/} . F A P^{-/-}$mice compared with their respective genotypes in male mice (Supplemental Figure S5I). The most striking effect of Fap deletion was on the prevalence of Mox macrophages, which were markedly decreased in female $A p o e^{-/-}$. Fap $^{-/-}$mice compared with female $\mathrm{Apoe}^{-/-}$mice or male Apoe ${ }^{-/-}$. Fap $^{-/-}$mice (Figure 9F). These data indicate that Fap deletion also affects the inflammatory response in a sexually dimorphic manner by reducing the accumulation of M1 and Mox macrophages in females.

\section{Discussion}

In summary, this study builds on the very limited data regarding FAP in CVD to show that Fap is expressed in lesions in a murine model of atherosclerosis, mirroring the findings in human disease. ${ }^{33,34}$ These reports showed that FAP expression in human lesions increased with plaque severity. In the $A p o e^{-/-}$mouse model this was not the case, with Fap expression following a normal distribution (during the time points examined), with peak expression observed at 30 weeks in males, whereas Fap expression was relatively consistently independent of time in females. These differences may be due to the experimental model. Indeed, although $\mathrm{Apoe}^{-/-}$mice and the well-used $\mathrm{Ldlr}^{--}$mouse are excellent models of atherosclerosis, they do not identically replicate the human disease. Notably, the arterial sites where they develop are not identical, and mice do not develop unstable plaques even in the face of severe hypercholesterolemia. ${ }^{53}$ The robust FAP expression observed suggested that the Apoe ${ }^{-/-}$model would act as an adequate surrogate for human disease despite these shortcomings.

The previous findings in human disease suggested that FAP expression was limited to VSMCs. ${ }^{34}$ It has been previously reported that Fap is robustly expressed in a subset of activated fibroblasts as well as the anti-inflammatory M2 subset of macrophages in the context of solid tumors. ${ }^{27}$ Both these cell types are localized to sites of atherosclerosis (fibroblasts being restricted to the adventitia); therefore, it was important to determine the cellular localization of Fap in the Apoe ${ }^{-/-}$mouse model. Co-immunostaining of Fap with the mesenchymal cell marker Vim (and not with the inflammatory cell marker Cd68) suggested that Fap was expressed on cells of mesenchymal origin in lesions.
Primary VSMCs examined in vitro revealed that Fap expression correlated with Vcam1 expression. Furthermore, Fap expression was inversely correlated with expression of Acta2, when stimulated with rhTGF- $\beta 1$. These data suggested that Fap was expressed on the subset of lesional dedifferentiated VSMCs rather than medial differentiated or lesional fibrotic VSMCs found in the vessel wall or fibrotic cap, respectively. Indeed, co-immunostaining of Fap with Acta2 in vivo clearly showed that $\mathrm{Fap}^{+}$- and $\mathrm{Acta}_{2}{ }^{+}$-stained regions in atherosclerosis were nonoverlapping. This finding is in concordance with previous data that showed that Fap ${ }^{+}$ and $\mathrm{Acta}^{+}$cell populations can be mutually exclusive or overlapping, depending on the pathological setting. ${ }^{54}$ Interestingly, co-immunostaining of Fap with the mesenchymal cell marker Vcam1 in vivo showed instances in which both proteins were co-localized and instances in which Fap was present in the absence of Vcam1 or vice versa. As discussed, lesional mesenchymal cells, represent a heterogeneous population in atherosclerosis, with lineage tracing studies showing that cells of VSMC origin can take on markers of inflammatory/macrophage-like cells, including Cd68 and LGALS3. ${ }^{11,41,42}$ Therefore, Fap may be identifying a previously uncharacterized subpopulation or transitional state of lesional dedifferentiated VSMCs. It is certainly possible that $\mathrm{Fap}^{+} \mathrm{Vcam}^{+}$versus $\mathrm{Fap}^{+} \mathrm{Vcam}^{-}$or $\mathrm{Fap}^{-} \mathrm{Vcam}^{+}{ }^{+}$lesional VSMCs have distinct characteristics that affect ECM deposition, remodeling, and cellular signaling in the lesional milieu. Going forward, it will be interesting to explore how these dedifferentiated subsets of VSMCs affect the progression of atherosclerosis.

Genetic deletion of Fap in Apoe ${ }^{-/-}$mice accelerated atherosclerosis in both males and females. This finding was in contrast to previous findings in cancer, where genetic deletion of Fap or targeted deletion of $\mathrm{Fap}^{+}$cells by T-cell immunotherapy significantly disrupted and slowed tumor progression. ${ }^{27,55}$ This finding suggests that therapeutic targeting of FAP for the treatment of atherosclerosis may not be suitable. The targeted deletion of $\mathrm{FAP}^{+}$cells may differently alter the progression of atherosclerosis, hypothetically by reducing dedifferentiated neointimal mesenchymal cell populations and therefore reducing lesion burden. However, a reduction in the number of lesional dedifferentiated mesenchymal cells may have the adverse effect of tipping the counterbalance of lesion content toward inflammation, therefore increasing the risk of plaque rupture leading to a myocardial event. In any case, this finding highlights that due care needs to be taken when therapeutically targeting FAP for certain diseases ${ }^{56}$ because it may lead to adverse complications in other pathological settings.

It was next determined if the increased atherosclerosis observed in $\mathrm{Apoe}^{-/-} . \mathrm{Fap}^{-/-}$mice was coupled with morphologic changes in lesional cellular make-up and/or ECM architecture. In Apoe ${ }^{-1-}$ mice, levels for most of the markers examined were comparable between males with the exception of M1 and M2 macrophages (M1s were slightly lower in females, whereas M2s were slightly higher). 
However, in many instances, the levels of markers between sexes in $\mathrm{Apoe}^{-/-} . \mathrm{Fap}^{-/-}$mice were different. Total collagen area, fibrillar collagen area/intensity, Col1 intensity, fibrotic cap length, and Fn1 intensity were higher in female $\mathrm{Apoe}^{-/-} . \mathrm{Fap}^{-/-}$mice compared with males. Conversely, lipid content, total Fn1 area, Adgre1 content, Cd68 content, M1 macrophage content, and Mox macrophage content were all lower in female $\mathrm{Apoe}^{-1-} . \mathrm{Fap}^{-1-}$ mice compared with males. Although many of these differences were modest (yet statistically significant), these combined data show that Fap has sexually dimorphic roles, may play a greater role in cellular mechanisms in females compared with males, and targeting Fap in females may have sex-specific effects. To determine the net effect of deletion of Fap on lesional morphology within each sex, a composition profile for each cohort was created (Table 2). In males, deletion of Fap accelerated atherosclerosis without significantly altering lesion composition; only minor decreases were observed in Coll and Acta2 content. In contrast, deletion of Fap in females also accelerated atherosclerosis that was accompanied with marked changes in ECM composition and inflammation. Col1-rich areas were increased coupled with a decrease in Fn1-rich regions. Furthermore, total macrophage content and specifically Mox macrophages were significantly decreased. Interestingly, the expression of Hmox 1 (the marker for Mox macrophages) is generally thought to negate vascular inflammation and foam cell formation and protect against atherogenesis. ${ }^{57}$ It has also been reported that a subset of M2 macrophages mediate immune suppression in tumors via the Hmox 1 pathway. ${ }^{58}$ Little to no M2 macrophages were observed within lesions in this study, and they were only detected sporadically in the adventitia. Although adventitial M2 macrophages may play an important role, any disease outcomes in this instance mediated via Fap and Hmox1 are more likely to arise from VSMC-Mox macrophage crosstalk. This study postulates that lesional Fap expression and the generation of collagen fragments is creating an inflammatory environment for the recruitment and retention of Mox macrophages. Curiously, this Fap-dependent inflammatory milieu may be antiatherogenic and a critical step in restraining atherosclerosis because deletion of Fap increased lesion size coupled with a decrease in Mox macrophage content. This hypothesis opposes previous findings in which expression of the elastase Mmp12 on Mox macrophages promoted atherosclerosis, ${ }^{10}$ suggesting that reduced accumulation of these cells may attenuate the disease. This finding can be explained as deletion of Fap also increasing the fibrotic content of lesions, which in turn overcomes the attenuated atherosclerotic phenotype observed by reducing levels of Mmp12 and leads to a net increase in lesion burden. Interestingly, a shift in the balance to lesional fibrosis versus inflammation (as we observed in Apoe $^{-1-}$.Fap $^{-1-}$ female mice) alternatively supports disease outcome by favoring plaque stability. Whether Fap is directly involved in cellular processes in both compartments and affects changes in cellular crosstalk directed from one cell population to the other is a complex question for future studies. Nevertheless, it will be critically important going forward to understand the net effect of targeting Fap on both the fibrotic and inflammatory responses to best determine its long-term effects on the disease.

These findings clearly show the existence of sexually dimorphic Fap-dependent mechanisms in atherosclerosis. Regarding sex, it is well established that sex hormones are intimately involved in the pathogenesis of atherosclerosis. There is a strikingly lower incidence of myocardial infarction in premenopausal women than men of the same age, followed by a dramatic increase in coronary risk after menopause. ${ }^{59,60}$ Indeed, estrogen is involved in the behavior of the major cell populations in atherosclerosis, including endothelial cells, VSMCs, macrophages, and dendritic cells. ${ }^{61}$ Generally speaking, estrogen signaling is considered to be antiatherogenic by promoting vasorelaxation via increased production of nitric oxide and $\mathrm{PGl}_{2}$, decreased expression of the adhesion molecules Vcam1 and intercellular adhesion molecule 1 , and increased proliferation in endothelial cells. Furthermore, its antiatherogenic effects extend to the neointimal cells, reducing Cd36-depedent cholesterol ingestion and inflammatory cytokine production (tumor necrosis factor- $\alpha$, monocyte chemoattractant protein-1, and NF- $\kappa \mathrm{B}$ among others) in macrophages. ${ }^{61}$ Antiatherogenic effects on VSMCs have also been established, including decreased proliferation and migration, reduced reactive oxygen species production, and production of the proinflammatory cytokines monocyte chemoattractant protein-1 and endothelin-1. ${ }^{61,62}$ Because FAP is predominantly expressed by lesional dedifferentiated mesenchymal cells (purportedly VSMCs) in atherosclerosis, any estrogenFap-dependent differences seen in our study may hypothetically arise from this population. These findings suggest that Fap may be a novel negative regulator of estrogen signaling, particularly in VSMCs. In the absence of Fap, VSMC-estrogen-dependent antiatherogenic signaling is increased, leading to further decreased proinflammatory cytokine/reactive oxygen species production and subsequently reduced lesional inflammation. In addition, in the absence of Fap, proteolysis of fibrillar collagen may be impaired, leading to increased fibrosis and reduced collagen fragmentation and uptake, a known stimulant of inflammation. ${ }^{63}$ In light of the findings reported here, if would be interesting to more fully identify Fap-dependent mechanisms involved in the development of profibrotic/antiinflammatory lesions in females to develop new therapeutic strategies to potentially reduce the risk of plaque rupture and myocardial events.

\section{Supplemental Data}

Supplemental material for this article can be found at http://doi.org/10.1016/j.ajpath.2020.01.004. 


\section{References}

1. Roger VL, Go AS, Lloyd-Jones DM, Adams RJ, Berry JD, Brown TM, et al: Heart disease and stroke statistics-2011 update: a report from the American Heart Association. Circulation 2011, 123: e18-e209

2. Steppan J, Barodka V, Berkowitz DE, Nyhan D: Vascular stiffness and increased pulse pressure in the aging cardiovascular system. Cardiol Res Pract 2011, 2011:263585

3. Wang X, Keith JC Jr, Struthers AD, Feuerstein GZ: Assessment of arterial stiffness, a translational medicine biomarker system for evaluation of vascular risk. Cardiovasc Ther 2008, 26:214-223

4. Sakakura K, Nakano M, Otsuka F, Ladich E, Kolodgie FD, Virmani R: Pathophysiology of atherosclerosis plaque progression. Heart Lung Circ 2013, 22:399-411

5. Nahrendorf M, Swirski FK: Lifestyle effects on hematopoiesis and atherosclerosis. Circ Res 2015, 116:884-894

6. Duprez D, Cohn J: Arterial stiffness as a risk factor for coronary atherosclerosis. Curr Atheroscler Rep 2007, 9:139-144

7. Mitchell GF, Hwang SJ, Vasan RS, Larson MG, Pencina MJ, Hamburg NM, Vita JA, Levy D, Benjamin EJ: Arterial stiffness and cardiovascular events: the Framingham Heart Study. Circulation 2010, 121:505-511

8. Kothapalli D, Liu SL, Bae YH, Monslow J, Xu T, Hawthorne EA, Byfield FJ, Castagnino P, Rao S, Rader DJ, Puré E, Phillips MC, Lund-Katz S, Janmey PA, Assoian RK: Cardiovascular protection by ApoE and ApoE-HDL linked to suppression of ECM gene expression and arterial stiffening. Cell Rep 2012, 2:1259-1271

9. Huynh J, Nishimura N, Rana K, Peloquin JM, Califano JP, Montague CR, King MR, Schaffer CB, Reinhart-King CA: Agerelated intimal stiffening enhances endothelial permeability and leukocyte transmigration. Sci Transl Med 2011, 3:112ra122

10. Liu SL, Bajpai A, Hawthorne EA, Bae Y, Castagnino P, Monslow J, Puré E, Spiller KL, Assoian RK: Cardiovascular protection in females linked to estrogen-dependent inhibition of arterial stiffening and macrophage MMP12. JCI Insight 2019, 4:e122742

11. Bennett MR, Sinha S, Owens GK: Vascular smooth muscle cells in atherosclerosis. Circ Res 2016, 118:692-702

12. Newby AC: Dual role of matrix metalloproteinases (matrixins) in intimal thickening and atherosclerotic plaque rupture. Physiol Rev 2005, 85:1-31

13. Shipley JM, Wesselschmidt RL, Kobayashi DK, Ley TJ, Shapiro SD: Metalloelastase is required for macrophage-mediated proteolysis and matrix invasion in mice. Proc Natl Acad Sci U S A 1996, 93: 3942-3946

14. Johnson JL, Baker AH, Oka K, Chan L, Newby AC, Jackson CL, George SJ: Suppression of atherosclerotic plaque progression and instability by tissue inhibitor of metalloproteinase-2: involvement of macrophage migration and apoptosis. Circulation 2006, 113: 2435-2444

15. Pepper MS: Role of the matrix metalloproteinase and plasminogen activator-plasmin systems in angiogenesis. Arterioscler Thromb Vasc Biol 2001, 21:1104-1117

16. Newby AC: Matrix metalloproteinases regulate migration, proliferation, and death of vascular smooth muscle cells by degrading matrix and non-matrix substrates. Cardiovasc Res 2006, 69:614-624

17. Park JE, Lenter MC, Zimmermann RN, Garin-Chesa P, Old LJ, Rettig WJ: Fibroblast activation protein, a dual specificity serine protease expressed in reactive human tumor stromal fibroblasts. J Biol Chem 1999, 274:36505-36512

18. Levy MT, McCaughan GW, Abbott CA, Park JE, Cunningham AM, Müller E, Rettig WJ, Gorrell MD: Fibroblast activation protein: a cell surface dipeptidyl peptidase and gelatinase expressed by stellate cells at the tissue remodelling interface in human cirrhosis. Hepatology 1999, 29:1768-1778
19. Acharya PS, Zukas A, Chandan V, Katzenstein AL, Puré E: Fibroblast activation protein: a serine protease expressed at the remodeling interface in idiopathic pulmonary fibrosis. Hum Pathol 2006, 37:352-360

20. Rettig WJ, Garin-Chesa P, Healey JH, Su SL, Ozer HL, Schwab M, Albino AP, Old LJ: Regulation and heteromeric structure of the fibroblast activation protein in normal and transformed cells of mesenchymal and neuroectodermal origin. Cancer Res 1993, 53: $3327-3335$

21. Aertgeerts K, Levin I, Shi L, Snell GP, Jennings A, Prasad GS, Zhang Y, Kraus ML, Salakian S, Sridhar V, Wijnands R, Tennant MG: Structural and kinetic analysis of the substrate specificity of human fibroblast activation protein alpha. J Biol Chem 2005, 280:19441-19444

22. Edosada CY, Quan C, Tran T, Pham V, Wiesmann C, Fairbrother W, Wolf BB: Peptide substrate profiling defines fibroblast activation protein as an endopeptidase of strict Gly2-Pro1-cleaving specificity. FEBS Lett 2006, 580:1581-1586

23. Aggarwal S, Brennen WN, Kole TP, Schneider E, Topaloglu O, Yates M, Cotter RJ, Denmeade SR: Fibroblast activation protein peptide substrates identified from human collagen I derived gelatin cleavage sites. Biochemistry 2008, 47:1076-1086

24. Tillmanns J, Hoffmann D, Habbaba Y, Schmitto JD, Sedding D, Fraccarollo D, Galuppo P, Bauersachs J: Fibroblast activation protein alpha expression identifies activated fibroblasts after myocardial infarction. J Mol Cell Cardiol 2015, 87:194-203

25. Bauer S, Jendro MC, Wadle A, Kleber S, Stenner F, Dinser R, Reich A, Faccin E, Gödde S, Dinges H, Müller Ladner U, Renner C: Fibroblast activation protein is expressed by rheumatoid myofibroblast-like synoviocytes. Arthritis Res Ther 2006, 8:R171

26. Garin-Chesa P, Old LJ, Rettig WJ: Cell surface glycoprotein of reactive stromal fibroblasts as a potential antibody target in human epithelial cancers. Proc Natl Acad Sci U S A 1990, 87: $7235-7239$

27. Lo A, Wang LCS, Scholler J, Monslow J, Avery D, Newick K, O'Brien S, Evans RA, Bajor DJ, Clendenin C, Durham AC, Buza EL, Vonderheide RH, June CH, Albelda SM, Puré E: Tumor-promoting desmoplasia is disrupted by depleting FAP-expressing stromal cells. Cancer Res 2015, 75:2800-2810

28. Tchou J, Zhang PJ, Bi Y, Satija C, Marjumdar R, Stephen TL, Lo A, Chen H, Mies C, June CH, Conejo Garcia J, Puré E: Fibroblast activation protein expression by stromal cells and tumor-associated macrophages in human breast cancer. Hum Pathol 2013, 44:2549-2557

29. Busek P, Balaziova E, Matrasova I, Hilser M, Tomas R, Syrucek M, Zemanova Z, Krepela E, Belacek J, Sedo A: Fibroblast activation protein alpha is expressed by transformed and stromal cells and is associated with mesenchymal features in glioblastoma. Tumor Biol 2016, 237:13961-13971

30. Yang X, Lin Y, Shi Y, Li B, Liu W, Yin W, Dang Y, Chu Y, Fan J, He R: FAP promotes immunosuppression by cancer-associated fibroblasts in the tumor microenvironment via STAT3-CCL2 signaling. Cancer Res 2016, 76:4124LP-4135LP

31. Kraman M, Bambrough PJ, Arnold JN, Roberts EW, Magiera L, Jones JO, Gopinathan A, Tuveson DA, Fearon DT: Suppression of antitumor immunity by stromal cells expressing fibroblast activation protein $-\alpha$. Science 2010, 330:827-830

32. Wang LCS, Lo A, Scholler J, Sun J, Majumdar RS, Kapoor V, Antzis M, Cotner CE, Johnson LA, Durham AC, Solomides CC, June $\mathrm{CH}$, Puré E, Albelda SM: Targeting fibroblast activation protein in tumor stroma with chimeric antigen receptor $\mathrm{T}$ cells can inhibit tumor growth and augment host immunity without severe toxicity. Cancer Immunol Res 2014, 2:154LP-166LP

33. Brokopp CE, Schoenauer R, Richards P, Bauer S, Lohmann C, Emmert MY, Weber B, Winnik S, Aikawa E, Graves K, Genoni M, Vogt P, Lüscher TF, Renner C, Hoerstrup SP, Matter CM: Fibroblast activation protein is induced by inflammation and degrades type I collagen in thin-cap fibroatheromata. Eur Heart J 2011, 32:2713-2722 
34. Meletta R, Müller Herde A, Chiotellis A, Isa M, Rancic Z, Borel N, Ametamey SM, Krämer SD, Schibli R: Evaluation of the radiolabeled boronic acid-based FAP inhibitor MIP-1232 for atherosclerotic plaque imaging. Molecules 2015, 20:2081-2099

35. Zhao L, Lee E, Zukas AM, Middleton MK, Kinder M, Acharya PS, Hall JA, Rader DJ, Puré E: CD44 expressed on both bone marrowderived and non-bone marrow-derived cells promotes atherogenesis in ApoE-deficient mice. Arterioscler Thromb Vasc Biol 2008, 28 : $1283-1289$

36. Cuff CA, Kothapalli D, Azonobi I, Chun S, Zhang Y, Belkin R, Yeh C, Secreto A, Assoian RK, Rader DJ, Puré E: The adhesion receptor CD44 promotes atherosclerosis by mediating inflammatory cell recruitment and vascular cell activation. J Clin Invest 2001, 108: $1031-1040$

37. Tang SY, Monslow J, Todd L, Lawson J, Puré E, FitzGerald GA: Cyclooxygenase-2 in endothelial and vascular smooth muscle cells restrains atherogenesis in hyperlipidemic mice. Circulation 2014, 129:1761-1769

38. Niedermeyer J, Kriz M, Hilberg F, Garin-Chesa P, Bamberger U, Lenter MC, Park J, Viertel B, Puschner H, Mauz M, Rettig WJ, Schnapp A: Targeted disruption of mouse fibroblast activation protein. Mol Cell Biol 2000, 20:1089-1094

39. Committee for the Update of the Guide for the Care and Use of Laboratory AnimalsNational Research Council: Guide for the Care and Use of Laboratory Animals: Eighth Edition. Washington, DC, National Academies Press, 2011

40. Zhu L, Stalker TJ, Fong KP, Jiang H, Tran A, Crichton I, Lee EK, Neeves KB, Maloney SF, Kikutani H, Kumanogoh A, Pure E, Diamond SL, Brass LF: Disruption of SEMA4D ameliorates platelet hypersensitivity in dyslipidemia and confers protection against the development of atherosclerosis. Arterio Thromb Vasc Biol 2009, 29: 1039-1045

41. Gomez D, Owens GK: Smooth muscle cell phenotypic switching in atherosclerosis. Cardiovasc Res 2012, 95:156-164

42. Harman JL, Jørgensen HF: The role of smooth muscle cells in plaque stability: therapeutic targeting potential. Br J Pharmacol 2019, 176 : $3741-3753$

43. Hastings NE, Feaver RE, Lee MY, Wamhoff BR, Blackman BR: Human IL-8 regulates smooth muscle cell VCAM-1 expression in response to endothelial cells exposed to atheroprone flow. Arterioscler Thromb Vasc Biol 2009, 29:725-731

44. Cybulsky MI, Iiyama K, Li H, Zhu S, Chen M, Iiyama M, Davis V, Gutierrez-Ramos JC, Connelly PW, Milstone DS: A major role for VCAM-1, but not ICAM-1, in early atherosclerosis. J Clin Invest 2001, 107:1255-1262

45. Polyzos KA, Ovchinnikova O, Berg M, Baumgartner R, Agardh H, Pirault J, Gisterå A, Assinger A, Laguna-Fernandez A, Bäck M, Hansson GK, Ketelhuth DF: Inhibition of indoleamine 2,3dioxygenase promotes vascular inflammation and increases atherosclerosis in Apoe-/- mice. Cardiovasc Res 2015, 106: 295-302
46. Kasper HU, Schmidt A, Roessner A: Expression of the adhesion molecules ICAM, VCAM, and ELAM in the arteriosclerotic plaque. Gen Diagn Pathol 1996, 141:289-294

47. Liu H, Xia Y, Li B, Pan J, Lv M, Wang X, An F: Prolyl hydroxylase 3 overexpression accelerates the progression of atherosclerosis in ApoE-/- mice. Biochem Biophys Res Commun 2016, 473:99-106

48. Avery D, Govindaraju P, Jacob M, Todd L, Monslow J, Puré E: Extracellular matrix directs phenotypic heterogeneity of activated fibroblasts. Matrix Biol 2018, 67:90-106

49. Pardali E, Ten Dijke P: TGF $\beta$ signaling and cardiovascular diseases. Int J Biol Sci 2012, 8:195-213

50. Bubb KJ, Khambata RS, Ahluwalia A: Sexual dimorphism in rodent models of hypertension and atherosclerosis. Br J Pharmacol 2012, $167: 298-312$

51. Fan MH, Zhu Q, Li HH, Ra HJ, Majumdar S, Gulick DL, Jerome JA, Madsen DH, Christofidou-Solomidou M, Speicher DW, Bachovchin WW, Feghali-Bostwick C, Puré E: Fibroblast activation protein (FAP) accelerates collagen degradation and clearance from lungs in mice. J Biol Chem 2016, 291:8070-8089

52. Moore KJ, Sheedy FJ, Fisher EA: Macrophages in atherosclerosis: a dynamic balance. Nat Rev Immunol 2013, 13:709-721

53. Getz GS, Reardon CA: Use of mouse models in atherosclerosis research. Methods Mol Biol 2015, 1339:1-16

54. Jacob M, Chang L, Puré E: Fibroblast activation protein in remodeling tissues. Curr Mol Med 2012, 12:1220-1243

55. Santos AM, Jung J, Aziz N, Kissil JL, Puré E: Targeting fibroblast activation protein inhibits tumor stromagenesis and growth in mice. J Clin Invest 2009, 119:3613-3625

56. Busek P, Mateu R, Zubal M, Kotackova L, Sedo A: Targeting fibroblast activation protein in cancer: prospects and caveats. Front Biosci (Landmark Ed) 2018, 23:1933-1968

57. Araujo JA, Zhang M, Yin F: Heme oxygenase-1, oxidation, inflammation, and atherosclerosis. Front Pharmacol 2012, 3:119

58. Arnold JN, Magiera L, Kraman M, Fearon DT: Tumoral immune suppression by macrophages expressing fibroblast activation protein$\alpha$ and heme oxygenase-1. Cancer Immunol Res 2014, 2:121-126

59. Lerner DJ, Kannel WB: Patterns of coronary heart disease morbidity and mortality in the sexes: a 26-year follow-up of the Framingham population. Am Heart J 1986, 111:383-390

60. Pérez-López FR, Chedraui P, Gilbert JJ, Pérez-Roncero G: Cardiovascular risk in menopausal women and prevalent related co-morbid conditions: facing the post-Women's Health Initiative era. Fertil Steril 2009, 92:1171-1186

61. Nofer JR: Estrogens and atherosclerosis: insights from animal models and cell systems. J Mol Endocrinol 2012, 48:R13-R29

62. Dehaini H, Fardoun M, Abou-Saleh H, El-Yazbi A, Eid AA, Eid AH: Estrogen in vascular smooth muscle cells: a friend or a foe? Vascul Pharmacol 2018, 111:15-21

63. O'Reilly PJ, Gaggar A, Blalock JE: Interfering with extracellular matrix degradation to blunt inflammation. Curr Opin Pharmacol $2008,8: 242-248$ 